\title{
On (cosmological) singularity avoidance in loop quantum gravity
}

\author{
J Brunnemann ${ }^{1,2}$ and T Thiemann ${ }^{1,2}$ \\ ${ }^{1}$ MPI für Gravitationsphysik, Albert-Einstein-Institut, Am Mühlenberg 1, 14476 Potsdam, \\ Germany \\ ${ }^{2}$ Perimeter Institute for Theoretical Physics, 31 Caroline Street N, Waterloo, ON N2L 2Y5, \\ Canada \\ E-mail: jbrunnem@aei.mpg.de, jbrunnemann@perimeterinstitute.ca, thiemann@aei.mpg.de and \\ thiemann@perimeterinstitute.ca
}

Received 24 October 2005

Published 13 February 2006

Online at stacks.iop.org/CQG/23/1395

\begin{abstract}
Loop quantum cosmology (LQC), mainly due to Bojowald, is not the cosmological sector of loop quantum gravity (LQG). Rather, LQC consists of a truncation of the phase space of classical general relativity to spatially homogeneous situations which is then quantized by the methods of LQG. Thus, LQC is a quantum-mechanical toy model (finite number of degrees of freedom) for LQG (a genuine QFT with an infinite number of degrees of freedom) which provides important consistency checks. However, it is a non-trivial question whether the predictions of LQC are robust after switching on the inhomogeneous fluctuations present in full LQG. Two of the most spectacular findings of LQC are that: (1) the inverse scale factor is bounded from above on zero-volume eigenstates which hints at the avoidance of the local curvature singularity and (2) the quantum Einstein equations are nonsingular which hints at the avoidance of the global initial singularity. This rests on (1) a key technique developed for LQG and (2) the fact that there are no inhomogeneous excitations. We display the result of a calculation for LQG which proves that the (analogon of the) inverse scale factor, while densely defined, is not bounded from above on zero-volume eigenstates. Thus, in full LQG, if curvature singularity avoidance is realized, then not in this simple way. In fact, it turns out that the boundedness of the inverse scale factor is neither necessary nor sufficient for the curvature singularity avoidance and that non-singular evolution equations are neither necessary nor sufficient for initial singularity avoidance because none of these criteria are formulated in terms of observable quantities. After outlining what would be required, we present the results of a calculation for LQG which could be a first indication that our criteria at least for curvature singularity avoidance are satisfied in LQG.
\end{abstract}

PACS numbers: 04.60.-m, 04.60.Pp 


\section{Introduction}

Loop quantum gravity (LQG) is a candidate for a quantum field theory (QFT) in four dimensions which achieves the unification of the principles of quantum theory (QT) and general relativity (GR). This means that LQG implements the fundamental feature of GR, its background independence, in a quantum setting; see e.g. [1] for books and [2] for reviews.

To see how radical that ambition is, recall that ordinary QFT relies on a background spacetime $\left(M, g_{0}\right)$. Here $M$ is a differential manifold and $g_{0}$ is a prescribed background metric thereon. Ordinary QFT now axiomatically assumes causality with respect to $g_{0}$ : given two smeared field operators $\phi(f), \phi\left(f^{\prime}\right)$ such that the supports of the smearing functions $f, f^{\prime}$ are spacelike separated (the non-spacelike geodesics with respect to $g_{0}$ starting from $\operatorname{supp}(f)$ never hit $\operatorname{supp}\left(f^{\prime}\right)$ and vice versa) then the field operators must (anti)commute. Thus we see that the background metric $g_{0}$ pivotally finds its way into the very definition of the field algebra. Without $g_{0}$ we therefore do not even know what a quantum field is (see however [3] for an interesting reformulation of ordinary QFT which may get us rid of $g_{0}$ ).

The background $g_{0}$, however, is incompatible with GR, in particular Einstein's equations, which state that the metric $g$ is a dynamical field which cannot be prescribed but must be determined dynamically according to the matter energy momentum density. Hence, ordinary QFT neglects backreaction effects from matter on geometry (which is appropriate in scattering situations of negligible particle numbers). Worse, if $g$ becomes itself a quantum field, in particular if there is no background $g_{0}$ such that the fluctuations $g-g_{0}$ are small in an appropriate sense, then ordinary QFT fails to be a valid description of the physical processes ${ }^{3}$. It is precisely in those situations that a full fledged quantum theory of both matter and geometry must take over and it is almost granted that these situations arise for instance in the early universe.

Thus, the task of a quantum gravity theory must be to define a quantum field just on a differential manifold $M$ and not on a background spacetime $\left(M, g_{0}\right)$. This means that one has to throw many aspects of conventional QFT overboard. LQG is trying to precisely achieve this goal. As one might expect, given that already classical GR is a rather difficult classical field theory, this is a very hard enterprise, both conceptually and technically. Despite these difficulties, LQG has made steady progress over the past 15 years and we are getting closer and closer to being able to answer the question whether LQG really is a QFT of GR in the sense that (1) classical GR is the classical limit of LQG and (2) when the fluctuations of the quantum metric are small, then the framework of QFT on $\left(M, g_{0}\right)$ is recovered. In other words, if these questions could be answered affirmatively, then we would have a viable quantum gravity theory in front of us and we could start drawing conclusions and physical predictions from it and hopefully compare those with experiments.

However, even before we (hopefully) complete the construction of full LQG, it is possible and mandatory to test the theory by models which are conceptually and technically easier to handle and which still capture enough of the features of the full theory. To give an example, the hydrogen atom can be analysed just using quantum mechanics for the electrons orbiting a classical nucleus and one gets fantastically close to the experiment. Hence, this gives us an important first idea. However, the full problem of the hydrogen atom must certainly take into account the full QFT of the standard model $^{4}$ and only then one can determine the Feinstruktur

\footnotetext{
3 The alternative, to quantize the fluctuations themselves and to construct a graviton QFT on $g_{0}$, fails due to (1) the non-renormalizability of GR (see however [4] for ideas concerning a non-trivial UV fix point) and (2) in violent situations there may simply be no $g_{0}$ at all such that the fluctuations are small.

4 Even today that has not yet been done fully, usually one only considers QED effects but not e.g. the QCD physics of the proton.
} 
of the hydrogen atom energy spectrum such as the Zitterbewegung of the electron due to the interaction with the electromagnetic field.

It is remarkable and important that the predictions of the simple quantum-mechanical computation are confirmed by the full theory because a priori it could be that the full QED calculation completely changes the picture. Of course, in the case of the hydrogen atom one has access to experimental data and so this was not expected if QED has something to do with nature. However, in the case of the absence of data there is no reason why one should trust a toy model calculation at all. In particular, it is unclear why a quantum-mechanical calculation describing only a finite number of degrees of freedom will reproduce the QFT aspects of the full problem involving an infinite number of degrees of freedom. One hopes of course that the full problem will only yield corrections to the model, however, that is far from granted and must be proved rigorously.

As far as LQG is concerned, in recent years a new class of toy models has been constructed which was coined loop quantum cosmology (LQC). These developments are mainly due to Bojowald; see e.g. the beautiful review [5]. These models consider the truncation of the infinite-dimensional phase space of GR to spatially homogeneous situations which are finite dimensional. Such truncations have been considered before and are called minisuperspace models [6]. Traditionally they were quantized using the Schrödinger representation of the canonical commutation relations. What is new in Bojowald's work is that he employs a different representation of the canonical commutation relations. This might come as a surprise because the Stone von Neumann theorem gives us a uniqueness result concerning the representation theory of the Weyl algebra for finite-dimensional phase spaces. The catch is that the Stone von Neumann theorem assumes, among other things, (weak) continuity of the Weyl operators. Bojowald drops this assumption because in full LQG the unique [7] representation [8] is also discontinuous, hence this representation is a much better model for LQG than the usual Schrödinger representation. This kind of representation was previously discussed by e.g. Thirring [9] and while exotic is used e.g. in solid-state physics [10]. Moreover, using this one can circumvent negative norm states in Maxwell theory [11] or string theory which then does not lead to restrictions on the spacetime dimension [12].

A second important ingredient in Bojowald's work is a key technique discovered for full LQG in [13] which allows one to define certain operators densely on the LQG Hilbert space although their classical counterpart is ill defined when the metric becomes degenerate. Prime examples for such classically singular situations are cosmological big bang singularities. This key technique was used in [13] in order to give, for the first time, a mathematical meaning to the quantum Einstein equations in full LQG, mathematically speaking, the so-called WheelerDeWitt equations could be defined densely on the LQG Hilbert space. Moreover, these equations can be solved explicitly by following a constructive algorithm [14]. This works for arbitrary matter coupling [15] and was tested successfully in $2+1$ gravity [16]. For a modern version of this framework see [17]. A simpler setting in which this key technique was used is the construction of the length operator in full LQG [18]. This operator is also well (i.e. densely) defined, it is an unbounded but positive self-adjoint operator whose spectrum is entirely discrete.

Of course, due to the complexity of the full theory, LQG cannot be solved completely. It is precisely the virtue of toy models such as LQC that their mathematics is comparatively simple so that one can focus immediately on the conceptual analysis. Bojowald therefore could go very far in the quantization of the cosmological models although some important aspects such as the physical inner product are still missing. The most spectacular finding is that in LQC the 
operator corresponding to the inverse scale factor $(s)^{5}$ is (are) bounded from above $[19,20]$. This hints at the following: recall that in classical GR one characterizes singularities by global and local criteria [21]. The global criterion is the causal geodesic incompleteness of a (inextendible) spacetime. Equivalently, the Einstein equations on globally hyperbolic spactimes with manifolds diffeomorphic to $\mathbb{R} \times \sigma$ cannot be solved for all values of $t \in \mathbb{R}$. The local criterion is defined as the divergence of polynomial scalars built out of the Riemann tensor and is used to characterize a global singularity in more detail. The local criterion by itself is unsatisfactory because a spacetime can have a global singularity while all curvature scalars are regular. Note that both criteria are to be measured by an observer and in this sense are to be formulated in terms of observables (measurable quantities).

In classical cosmology both types of singularities are present. Since in cosmology curvature scalars are given by powers of the inverse scale factor, its boundedness hints at the absence of the local singularity in LQC in the sense that with respect to any kinematical state the curvature expectation value can never diverge. Moreover, the truncated quantum Einstein equations (Wheeler-DeWitt equation) do not become singular at the classical big bang and one therefore can go before the big bang. This hints at global singularity avoidance in LQC. Further consequences of the boundedness of the inverse scale factor are that the quantum geometry behaves very non-classically at the classical singularity implying effective modifications of the Friedmann equations which then drive geometric inflation without the necessity of fine tuning the inflaton potential [22]. These results related to the boundedness of the inverse scale factor in LQC are so promising and spectacular that it was extensively highlighted in the physics literature and public press. In particular, the boundedness was mentioned as crucial to establish the non-singularity of the quantum evolution equations; see e.g. [23].

In [24] it was shown that it is precisely those two ingredients imported from full LQG into LQC, (1) the non-standard representation of the canonical commutation relations and (2) the key technique [13] to densely define classically degenerate operators, which are responsible for the absence of the local singularity in LQC and similar models inspired more by a metric than connection-based approach. However, as outlined above, on general grounds one must critically examine the stability of such model calculations when one reintroduces the field theory degrees of freedom. This has been stressed before for geometrodynamics in [25] where one generically finds that the model even quantitatively has large deviations from the full theory or more complete theory. More recently also LQC has been examined critically [26] concerning the issue of the semiclassical limit but here the results seem to be inconclusive so far.

The main purpose of this paper is to contribute to the stability analysis of LQC versus LQG. The paper is organized as follows. In section 2, we summarize and compare the main features of LQG and LQC for the unfamiliar reader. Experts can safely skip this section. In section 3, we report the results of a computation carried out in our companion paper [28] within full LQG which is analogous to the one carried out in LQC. In LQC this calculation proves the boundedness of the inverse scale factor. More precisely, we ask, as in LQC, whether the part of the energy momentum operator which probes the quantum metric is bounded from above on the kinematical Hilbert space of full LQG, at least at the classical singularity, that is, when the volume of space vanishes. This precisely mirrors the calculation of [19]. The result is negative: in full LQG the (analogon of) the inverse scale factor is unbounded from above even when the quantum volume vanishes. This is true even when we compute its norm with respect to a state of zero volume which is homogeneous and isotropic on large scales. This

5 One for the isotropic models, three for the diagonal models. 
proves that the boundedness of the inverse scale factor in isotropic and homogeneous LQC does not extend to the full theory even when restricting LQG to those states which one would use to describe a maximally homogeneous and isotropic situation (modulo fluctuations).

It is true that in non-isotropic, homogeneous models the inverse scale factor is also unbounded on the full Hilbert space. However, in those more general models it is still bounded on zero-volume eigenstates which again seems to indicate that the local singularity is evaded in LQC. Our result is stronger: no matter whether homogeneous and/or isotropic, spherically symmetric, cylindrically symmetric etc, the inverse scale factor is unbounded on zero-volume eigenstates in full LQG and on the full Hilbert space anyway. The reason for this is that LQG, in contrast to LQC, admits inhomogeneous, microscopical excitations which precisely account for the unboundedness. We will see this explicitly when we construct the zero-volume eigenstates on which the inverse scale factor has arbitrarily large norm. Note that meanwhile also inhomogeneous models such as spherically symmetric or cylindrically symmetric ones have been quantized by LQG methods in which the inverse scale factor apparently also stays bounded [27]. While these do have local, inhomogeneous degrees of freedom (in the spherically symmetric case without matter, at least before solving the constraints), as our calculation reveals, the inhomogeneous excitations of full LQG are more general than in those models and thus give unboundedness even on states of LQG describing a sector appropriate for those more general models.

However, this does not mean that LQG does not predict the absence of the local initial singularity. Namely, in section 4 we remark that an operator may be unbounded but still it may be bounded when restricted to a subspace of the Hilbert space. Thus, the boundedness of the inverse scale factor is not a necessary criterion for local singularity avoidance. In our case we are interested in a sector which describes a collapsing universe which is homogeneous and isotropic at large scales. We perform a corresponding calculation which may be taken as an indication that the expectation value of the analogon of the inverse scale factor in LQG, with respect to kinematical ${ }^{6}$, coherent states [43] peaked on homogeneous and isotropic initial data, is bounded from above at the big bang ${ }^{7}$. We did this for scalar matter only but qualitatively nothing changes in more general situations. Also our result is completely general: we derive a bound of the inverse scale factor on coherent states peaked on an arbitrary point in phase space. This formula can then be specialized also to inhomogeneous models and for the Bianchi I (Kasner) case it is easy to see that one gets boundedness as well.

In section 5, we stress that the results of section 4 are promising but inconclusive: unfortunately, the boundedness of the inverse scale factor is also not a sufficient criterion for local singularity avoidance. This has to do with the fact that both singularity criteria fundamentally have to be discussed at the level of physical observables and physical states. They must be discussed separately because the quantum theory could avoid one of them but not the other.

Concerning the local singularity, the inverse scale factor is not gauge invariant and therefore not an observable. It can be turned into an observable using the technique of partial observables $[29,30]$ but then its spectrum on the physical Hilbert space can differ drastically from the kinematical spectrum [31]. Hence, reliable statements about the local singularity can only be obtained on the physical Hilbert space.

As far as the global singularity is concerned, physical states automatically solve the quantum Einstein equations so there seems to be no sign of the global singularity at the

\footnotetext{
6 Kinematical states, in contrast to physical states, do not solve the quantum Einstein equations. Non-gauge invariant operators such as the inverse scale factor can only be probed on the kinematical Hilbert space.

7 Note that coherent states peaked on homogeneous and isotropic initial data still have inhomogeneous and anisotropic excitations, however, they are small.
} 
level of physical states, by construction ${ }^{8}$. More precisely, in order to solve the quantum Einstein equations, one takes an arbitrary superposition of kinematical states and then obtains a recursion relation for the coefficients. These have been derived explicitly for full LQG in [14] and those of LQC [19] are similar but simpler. One of the labels of these coefficients can be identified with an unphysical time parameter. If one chooses that label in such a way that its range is (a discrete subset of) the full real axis, a solution to the quantum Einstein equations, that is, a physical state, describes an entire quantum universe (a history) and involves the full range of the unphysical time parameter. Thus there is no sign of the global singularity within a given solution even in the full theory. However, following [19] one may argue that the existence of a global singularity could manifest itself in the fact that the recursion relations break down at zero volume. Such a breakdown can manifest itself in the following two ways:

A. It can lead to a restriction or additional consistency condition on the freedom in the choice of the initial data (coefficients) that parametrize the solution space. This makes the solution space smaller than it would be otherwise. In particular, if the number of additional conditions is larger than the free initial parameters of the solution, the solution space would be empty.

B. It can happen that certain coefficients remain undetermined by the recursion relation and lead to an indeterministic quantum evolution (given initial coefficients, we cannot construct the full solution without making additional choices). This makes the solution space larger than it would be otherwise. Note that this undeterministic quantum evolution corresponds to an undeterministic classical evolution (initial value problem). However, by itself such ambiguities are not physically relevant because this is not an evolution of physical quantities. Even classically it is an evolution with respect to an unphysical time of unphysical quantities. In terms of physical (gauge invariant) quantities there is no unphysical evolution at all by definition because Dirac observables Poisson commute with the Hamiltonian constraint. Hence, what we actually have to do in order to observe a possible breakdown of evolution is to consider an evolution with respect to a physical Hamiltonian and of physical observables. We will discuss this at length in section 5.

We now point out that the absence of either effect A or B is neither a sufficient nor a necessary criterion for global singularity avoidance.

To see that it is not necessary, note that the presence of either effect indeed affects the size of the set of formal solutions to the evolution equations. However, even if such effects exist, it may still be the case that the physical Hilbert space is large enough in order to accommodate all semiclassical physical states which describe all classical spacetimes (at large scales). To see that it is not sufficient, note that not all formal solutions to the quantum Einstein equations correspond to physical states because they might not be normalizable with respect to the physical inner product or they might have zero norm. In both cases we must drop them from the set of physical states. Hence, even if effect A or B is absent, it could turn out, in the worst case, that most of the solutions that one finds do not lie in the physical Hilbert space and then again one would be confronted with a global singularity because there could be not even be a semiclassical sector describing cosmology. Furthermore, as argued above, global singularity avoidance must be formulated in terms of physical observables which has not been done yet. Therefore, the breakdown of the recursion or the absence thereof is inconclusive for the presence or absence of global singularities unless one knows the physical Hilbert space and the corresponding Dirac observables.

8 Of course, the inverse scale factor must be densely defined to even define the quantum Einstein equations but that has been established for LQG in $[13,15]$. 
We conclude that in order to decide on the presence or absence of both the local and global singularities, detailed knowledge of the physical Hibert space is a necessary prerequisite. This Hilbert space is known to exist both in LQG and LQC, following for instance [17]. However, the corresponding physical inner product is rather difficult to construct explicitly, for any of the current versions of the Hamiltonian constraint, which is why a definitive conclusion on the singularity issue is not available at the moment.

To improve on this, we first of all broadly outline which steps should be fundamentally performed in order to prove the absence of both the global and local big bang singularity in full LQG. We give both a precise and an approximate scheme. The ideal (precise) procedure involves the exact knowledge of the Hilbert space of physical states and a sufficiently large set of Dirac observables (gauge invariant quantities) which, as we said, are not yet available neither in full LQG nor in LQC in a form explicit enough. The approximate scheme stays within the kinematical Hilbert space over which one has excellent control. The calculation performed in section 4 can be viewed as a simplified version of the approximate scheme. The application of either scheme is left for future research and again LQC would be an ideal testing ground for this scheme.

We conclude in section 6 summarizing the status of the absence of the initial singularity in full LQG. Note that qualitatively all we have said is also applicable to other singularities such as those connected with black holes [32, 33]. In fact, most considerations within LQG concerning black holes are based on minisuperspace calculations which exploit the fact that the interior of a black hole can be mapped into a homogeneous Kantowski-Sachs model so that one can transfer, almost literally, the LQC results. However, our remarks prevail: no reliable conclusions can be drawn before one works with physical operators and physical states of the full theory. This has also been stressed in [33].

Note that this paper is not meant as a criticism of LQC by itself, on the contrary, LQC has provided us with many fruitful new conceptual ideas and one would hope that its results hold also for LQG, albeit in a technically different realization. However, we want to draw attention to the fact that LQC is far from being full LQG and that therefore caution should be applied when trying to extrapolate LQC results to LQG. This extends also to more general reduced models such as the spherically symmetric or cylindrically symmetric ones.

\section{Relation between LQG and LQC}

For the benefit of the reader unfamiliar with the basic structure of either LQG or LQC we here summarize elements of both frameworks and compare them. The presentation will be oversimplified and the mathematical details will be discarded. As we will see, LQC does not describe a stable subsector of LQG, rather it is a truncation in which all but finitely many degrees of freedom are cancelled by hand. Experts can safely skip this section.

\subsection{Elements of $L Q G$}

The classical starting point of LQG is a Hamiltonian formulation of the Einstein-Hilbert action, however, written in unusual variables. Instead of using the three metric $q_{a b}$ on the spatial slices $\sigma$ of a foliation of the four manifold $M \cong \mathbb{R} \times \sigma$ and the extrinsic curvature $K_{a b}$ as the canonical variables as advocated by Arnowitt, Deser and Misner, one uses variables that are more familiar from the canonical formulation of Yang-Mills theories. They consist of an $S U(2)$ connection $A_{a}^{j}$ and an electric field $E_{j}^{a}$. Here $a, b, c, \ldots=1,2,3$ are tensorial indices and $j, k, l, \ldots=1,2,3$ are $s u(2)$ indices. The origin of $S U(2)$ is that $S U(2)$ is the universal covering group of $S O(3)$ (necessary for coupling of spinorial matter) and $S O(3)$ 
appears naturally when we write the three metric in terms of cotriads (or frame fields) $e_{a}^{j}$, that is, $q_{a b}=e_{a}^{j} e_{b}^{k} \delta_{j k}$. Indeed, the cotriads are determined by $q_{a b}$ only up to an $S O(3)$ rotation and this is how $S U(2)$ finds its way into this formulation as a gauge group.

The relation between $(A, E)$ and $(q, K)$ is

$$
A_{a}^{j}=\Gamma_{a}^{j}+\beta K_{a b} e_{j}^{b}, \quad E_{j}^{a}=\sqrt{\operatorname{det}(q)} e_{j}^{a} .
$$

Here $\Gamma$ is the spin connection (a certain function of $e_{a}^{j}$ and its first spatial derivatives), the triad $e_{j}^{a}$ is the inverse of $e_{a}^{j}$, i.e. $e_{j}^{a} e_{b}^{j}=\delta_{b}^{a}, e_{j}^{a} e_{a}^{k}=\delta_{j}^{k}$ and $\beta>0$ is a parameter called the Immirzi parameter. That $(A, E)$ form a canonical pair means that

$$
\left\{E_{j}^{a}(x), A_{b}^{k}(y)\right\}=8 \pi G \beta \delta_{b}^{a} \delta_{j}^{k} \delta(x, y),
$$

all others vanishing. The phase-space coordinatized by $(A, E)$ is subject to the usual spatial diffeomorphism constraint which in these variables can be written as

$$
C_{a}=F_{a b}^{j} E_{j}^{b} \quad \text { where } \quad F_{a b}^{j}=\partial_{a} A_{b}^{j}-\partial_{b} A_{a}^{j}+\epsilon_{j k l} A_{a}^{k} A_{b}^{l}
$$

is the curvature of $A$, the Hamiltonian constraint

$$
C=\frac{\epsilon_{j k l}\left[F_{a b}^{j}-\left(1+\beta^{2}\right) \epsilon_{j m n} K_{a}^{m} K_{b}^{n}\right] E_{k}^{a} E_{l}^{b}}{\sqrt{|\operatorname{det}(E)|}}
$$

where $K_{a}^{j}=A_{a}^{j}-\Gamma_{a}^{j}(E)$ and the additional Gauss constraint

$$
C_{j}=\partial_{a} E_{j}^{a}+\epsilon_{j k l} A_{a}^{k} E_{l}^{a},
$$

which gets us rid of the $S U$ (2) degrees of freedom. We see that GR can be cast into the form of a $S U$ (2) Yang-Mills theory (which is also subject to $C_{j}=0$ ) with one important difference: the background dependent Yang-Mills-Hamiltonian density

$$
H=\frac{q_{a b}^{0}}{\sqrt{\operatorname{det}\left(q^{0}\right)}}\left[E_{j}^{a} E_{k}^{b}+B_{j}^{a} B_{k}^{b}\right] \delta^{j k}
$$

where $B_{j}^{a}=\epsilon^{a b c} F_{b c}^{j} / 2$ is the magnetic field and $q^{0}$ is the spatial projection of the background metric $g^{0}$, is replaced by the background-independent constraints $C_{a}, C$.

If there is matter present such as a scalar field $\phi$ then the constraints $C_{a}, C$ are augmented by additional terms

$$
C_{a}^{\text {scalar }}=\pi \phi_{, a} \quad \text { and } \quad C^{\text {scalar }}=\frac{1}{2} \frac{\pi^{2}+E_{j}^{a} E_{j}^{b} \phi_{, a} \phi_{, b}}{\sqrt{|\operatorname{det}(E)|}}+\sqrt{|\operatorname{det}(E)|} V(\phi),
$$

where $\pi$ is the momentum conjugate to $\phi$.

Canonical quantization now, very roughly, means to find a Hilbert space $\mathcal{H}_{\text {kin }}$ on which the canonical Poisson brackets (2.2) are implemented as canonical commutation relations and to impose the quantum constraints, e.g. $\hat{C} \psi=0$. The resulting space of solutions is the physical Hilbert space on which the (Dirac) observables, that is, the gauge invariant functions $F$ on phase space satisfying $\left\{C_{a}(x), F\right\}=\{C(x), F\}=\left\{C_{j}(x), F\right\}=0$ act as self-adjoint operators $\hat{F}$. This simple recipe has to be refined in QFT because of the singular short distance behaviour of the fields $A, E$. In order to deal with this problem one has to smear the fields. There are many ways to smear the fields, each of which gives rise to a different algebra $\mathfrak{A}$ of elementary observables ${ }^{9}$. Guided by experience with canonical lattice gauge field theory in LQG one considers the following objects:

9 They are called elementary because one can write every function on phase space in terms of (limits of) them. They are not physically observable because they are not gauge invariant. 
1. For each curve $e$ in $\sigma$ the holonomy of the connection $A$ along $e$, that is,

$$
A(e):=\mathcal{P} \exp \left(\int_{e} A\right):=1_{2}+\sum_{n=1}^{\infty} \int_{0}^{1} \mathrm{~d} t_{1} \int_{t_{1}}^{1} \mathrm{~d} t_{2} \cdots \int_{t_{n-1}}^{1} \mathrm{~d} t_{n} A\left(t_{1}\right) \cdots A\left(t_{n}\right),
$$

where $e:[0,1] \rightarrow e ; t \mapsto e(t)$ is a parametrization of the curve and $A(t):=$ $A_{a}^{j}(e(t)) \dot{e}^{a}(t) \tau_{j}$ with $\tau_{j}=-\mathrm{i} \sigma_{j} / 2$ a basis of $s u(2)\left(\sigma_{j}\right.$ are the Pauli matrices).

2. For each surface $S$ and each $s u(2)$ valued function $x \mapsto f^{j}(x)$ the electric flux of $E$ through $S$

$$
E_{f}(S)=\int_{s} \mathrm{~d}^{2} u f^{j}\left(X_{S}(u)\right) \epsilon_{a b c} E_{j}^{a}\left(X_{S}(u)\right) X_{S, u^{1}}^{b}(u) X_{S, u^{2}}^{c}(u),
$$

where $X_{S}: s \subset \mathbb{R}^{2} \rightarrow S ; u=\left(u^{1}, u^{2}\right) \mapsto X_{S}(u)$ is a parametrization of $S$.

Using (2.2) one can show that the Poisson brackets ${ }^{10}$ of the holonomies and fluxes close among each other so that they define a well-defined algebra $\mathfrak{A}$.

The kinematical Hilbert space of LQG can now be described very easily as follows: a union of curves $e$ forms a graph $\gamma$. By subdividing the curves we may assume that the curves are mutually disjoint except for the endpoints. These curves are then called edges of $\gamma$ which intersect in the vertices. We will denote by $E(\gamma), V(\gamma)$ the set of edges and vertices of a graph respectively. The wavefunctions are then of the form

$$
\psi(A)=\psi_{\gamma}\left(A\left(e_{1}\right), \ldots, A\left(e_{N}\right)\right),
$$

where $\gamma$ can be any graph, $N$ is the number of edges of the graph $\gamma$ and $f_{\gamma}: S U(2)^{N} \rightarrow \mathbb{C}$ is a complex-valued function on $N$ copies of $S U(2)$.

The holonomy operator acts by multiplication

$$
[\widehat{A(e)} \psi](A):=A(e) \psi(A),
$$

while the fluxes act by derivation

$$
\left[\widehat{E_{f}(S)} \psi\right](A):=\mathrm{i} \hbar\left\{E_{f}(S), \psi(A)\right\} .
$$

The scalar product between functions $\psi, \psi^{\prime}$ defined via (2.10) over graphs $\gamma, \gamma^{\prime}$ respectively is defined as follows: take the union graph $\gamma^{\prime \prime}:=\gamma \cup \gamma^{\prime}$ and write the edges $e, e^{\prime}$ of $\gamma, \gamma^{\prime}$ respectively as compositions of edges $e^{\prime \prime}$ of $\gamma^{\prime \prime}$, say $e=e_{1}^{\prime \prime} \circ \cdots \circ e_{n}^{\prime \prime}$. Now use the fact that the holonomy factorizes, e.g. $A(e)=A\left(e_{1}^{\prime \prime}\right) \cdots A\left(e_{n}^{\prime \prime}\right)$, to write $\psi, \psi^{\prime}$ as functions over $\gamma^{\prime \prime}$. Then

$$
\left\langle\psi, \psi^{\prime}\right\rangle:=\int_{S U(2)^{N}} \mathrm{~d} \mu_{H}\left(h_{1}\right) \cdots \mathrm{d} \mu_{H}\left(h_{N}\right) \overline{\psi_{\gamma^{\prime \prime}}\left(h_{1}, h_{N}\right)} \psi_{\gamma^{\prime \prime}}^{\prime}\left(h_{1}, \ldots, h_{N}\right),
$$

where $N$ is the number of edges of $\gamma^{\prime \prime}$ and $\mu_{H}$ is the Haar measure on $S U$ (2). Specifically, if we write $h=\cos (\chi) 1_{2}+\tau_{j} n^{j}(\theta, \varphi) \sin (\chi)$ with $\chi, \theta \in[0, \pi)$ and $\varphi \in[0,2 \pi)$ where $\vec{n}=(\sin (\theta) \cos (\varphi), \sin (\theta) \sin (\varphi), \cos (\theta))$ is the usual unit vector on the sphere then $\mathrm{d} \mu_{H}(h)=c \sin ^{2}(\chi) \sin (\theta) \mathrm{d} \chi \mathrm{d} \theta \mathrm{d} \varphi$ and $c$ is a normalization constant fixed by requiring that $\mu_{H}(S U(2))=1$.

It turns out that the Hilbert space $\mathcal{H}_{\text {kin }}$ has a convenient orthonormal basis consisting of the spin network functions (SNF) $T_{\gamma, \vec{j}, \vec{I}}$. For the purpose of this paper it is enough to say that they are specific functions of type (2.10) which are polynomials in the holonomies $A(e), e \in E(\gamma)$. They are labelled by a graph $\gamma$, a collection $\vec{j}:=\left\{j_{e}\right\}_{e \in E(\gamma)}$ of spin quantum numbers $j_{e}, 2 j_{e}=1,2,3, \ldots$, one for each edge, and a collection of other quantum numbers $\vec{I}=\left\{I_{v}\right\}_{v \in V(\gamma)}$ called intertwiners, one for each vertex. Since the label $\gamma$ is continuous, the basis is uncountable and the Hilbert space is therefore not separable. We will use the short-hand $s=(\gamma(s), \vec{j}(s), \vec{I}(s))$ for a spin network label.

\footnotetext{
${ }^{10}$ More precisely the corresponding Hamiltonian vector fields.
} 
Let us now turn to the quantum constraints. The Gauss constraint imposes certain restrictions on the quantum numbers $\vec{I}$ of the SNF and is easily taken care of. The spatial diffeomorphism constraint enforces that we should identify all spin network labels $s, s^{\prime}$ if there exists a spatial diffeomorphism $\varphi$ of $\sigma$ such that $\varphi(\gamma(s))=\gamma\left(s^{\prime}\right)$ and so is also easily taken care of. The real challenge for LQG (and any other quantum gravity theory) is the Hamiltonian constraint. Looking at (2.4) the mere definition of the operator $\hat{C}$ looks like a hopeless task due to the nonlinearity of the constraint. It is here where the key technique of [13] comes into play: it is easy to see that the nonlinear expression involving the electric field $E$ can be written in the compact Poisson bracket form

$e_{a}^{j}=\frac{\epsilon_{a b c} \epsilon^{j k l} E_{k}^{b} E_{l}^{c}}{\sqrt{|\operatorname{det}(E)|}} \propto\left\{A_{a}^{j}, V\right\} \quad$ where $\quad V=\int_{\sigma} \mathrm{d}^{3} x \sqrt{|\operatorname{det}(E)|}$,

is the volume ${ }^{11}$ of $\sigma$. The advantage of this way of writing (2.14) is that there exists a volume operator corresponding to $V$ which can be diagonalized in terms of spin network states. Thus, (2.14) can be quantized by substituting Poisson brackets by commutators divided by i $\hbar$. Next, consider the smeared quantity $C(N)=\int \mathrm{d}^{3} x N(x) C(x)$ where $N$ is a test function. We may write this as $C(N)=C_{F}(N)+C_{K}(N)$ where $C_{F}, C_{K}$ respectively correspond to the terms in (2.4) involving the term $F_{a b}^{j}$ or $K_{a}^{m} K_{b}^{n}$ respectively. Now it is easy to see that

$$
K_{a}^{j}(x) \propto\left\{A_{a}^{j}(x),\left\{C_{F}(1), V\right\}\right\},
$$

where $C_{F}(1):=C_{F}(N)_{N=1}$. It follows that we know how to quantize $C(N)$ once we know how to quantize $C_{F}(N) .{ }^{12}$ In order to do this it remains to write $C_{F}(N)$ in terms of holonomies. This is achieved by recalling that for a curve $e_{x, u}^{a}(t):=x^{a}+t u^{a}$ and loop $\alpha_{x, u, v}(\epsilon):=$ $e_{x, u}(\epsilon) \circ e_{x+\epsilon u, v}(\epsilon) \circ e_{x+\epsilon(u+v), u}(\epsilon)^{-1} \circ e_{x+\epsilon, v}(\epsilon)^{-1}$ respectively we have the expansions $A\left(e_{x, u}(\epsilon)\right)=1_{2}+\epsilon u^{a} A_{a}^{j}(x) \tau_{j} / 2+O\left(\epsilon^{2}\right)$ and $A\left(\alpha_{x, u, v}(\epsilon)\right)=1_{2}+\epsilon^{2} u^{a} v^{b} F_{a b}^{j}(x) \tau_{j} / 2+O\left(\epsilon^{3}\right)$. Therefore, if we write the integral $C_{F}(N)$ as the limit of a Riemann sum over boxes of coordinate volume $\epsilon^{3}$ then in the limit it is appropriate to replace $F_{a b}^{j}$ by a holonomy along a loop on the boundary of the box while the $A_{a}^{j}$ in the Poisson bracket $\left\{A_{a}^{j}, V\right\}$ may be replaced by a holonomy along an edge on the boundary of that box.

The details of taking that limit can be inferred from $[13,17]$. Roughly speaking, one defines (a spatially diffeomorphism invariant version of) the constraint on the space of solutions to the diffeomorphism constraint on which the limit becomes trivial because what matters to the constraint are only the terms of the Riemann sum next neighbouring the vertices and those terms are diffeomorphic to each other when we refine the Riemann sum.

\subsection{Elements of $L Q C$ and comparison with $L Q G$}

LQC, by definition, is the canonical quantization of the cosmological truncation of classical GR by the methods of $\mathrm{LQG}^{13}$. Hence one first specializes the functions $A_{a}^{j}(x), E_{j}^{a}(x)$ to the spatially homogeneous case, plugs those into the formulae for the holonomies and fluxes

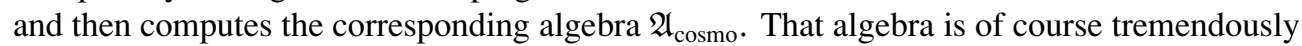
smaller than the full algebra $\mathfrak{A}$ but one quantizes it in analogy to LQG, that is, one chooses a Hilbert space representation which is as similar as possible to the one of LQG. Finally, one

${ }^{11}$ In the case of $k=0$ this diverges classically. However, we only need its Poisson bracket which is well defined.

12 The condition $C(x)=0$ for all $x$ is equivalent to requiring $C(N)=0$ for all $N$ because the support of $N$ can be localized arbitrarily.

${ }^{13}$ Originally one tried to define LQG as a symmetric sector of distributions on a dense sunspace of the LQG Hilbert space together with the dual action of the full LQG operators. However, this dual action does not preserve the symmetric sector. See also the end of this section. We adopt here the correct and modern point of view spelled out in [37]. 
plugs the simplified expression for $(A, E)$ into the constraints and quantizes them as in LQG, in particular one uses the technique (2.14) which enables one to avoid denominators (which are singular at the classical singularity) at the price of employing Poisson brackets with the volume functional.

Let us see this in more detail ${ }^{14}$ : we will consider the simplest case. The isotropic, flat FRW cosmologies for which the line element reads

$$
\mathrm{d} s^{2}=-\mathrm{d} t^{2}+a(t)^{2} \mathrm{~d} \vec{x}^{2},
$$

where $a$ is the scale factor. Here we take $t, a$ to have dimensions of length. One fixes both the spatial diffeomorphism constraint and the Gauss constraint by setting

$$
A_{a}^{j}:=q \delta_{a}^{j} / 3, \quad E_{j}^{a}:=p \delta_{j}^{a},
$$

where $p, q$ are spatial constants. It follows that $a=\sqrt{|p|}$ and that $\{p, q\}=8 \pi \beta G$ are canonically conjugate. It is easy to see that with (2.17) both the Gauss and spatial diffeomorphism constraint vanish identically as they should and that the gravitational contribution to the Hamiltonian constraint becomes $C=-\alpha q^{2} \sqrt{|p|}$ with a positive constant $\alpha$. For a (homogeneous) scalar field we find

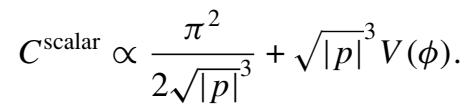

As one can show (see the end of section 4) the classical singularity is encoded in the kinetic term only.

The holonomy and flux become respectively

$$
A(e)=\cos \left(q r_{e}\right)+\tau_{j} n_{e}^{j} \sin \left(r_{e} q\right), \quad E_{f}(S)=\left[\int_{S} \mathrm{~d} s_{a} f^{a}\right] p,
$$

where $e^{a}(1)-e^{a}(0)=: r_{e} n_{e}^{j},\left(n_{e}^{j}\right)^{2}=1$. By varying $e$ and taking traces we may generate the functions $T_{r}:=\exp (\mathrm{i} q q)$ where $r$ can be any real number. The reduced holonomy flux algebra therefore is generated by the $T_{r}$ and $p$.

The reduced Hilbert space is now obtained by asking that $T_{r}$ form an orthonormal basis, i.e. $\left\langle T_{r}, T_{r^{\prime}}\right\rangle=\delta_{r, r^{\prime}}$ so that they play the analogon of the SNF. Here the continuous graph label and the discrete spin label of the SNF have joined into one continuous label $r$. The operators $\widehat{T}_{r}, \hat{p}$ act by multiplication and derivation respectively, explicitly $\widehat{T}_{r} T_{r^{\prime}}=T_{r+r^{\prime}}$ and $\hat{p} T_{r}:=\mathrm{i} \hbar\left\{p, T_{r}\right\}=-r \ell_{p}^{2} \beta T_{r}$ where $\ell_{p}^{2}=8 \pi G \hbar$ is the Planck length squared.

Finally, in order to quantize objects like the inverse scale factor $1 / a=1 / \sqrt{|p|}$ we note the classical identity

$$
\frac{1}{\sqrt{|p|}} \propto \frac{T_{r_{0}}\left\{T_{-r_{0}}, \sqrt{|p|}\right\}-T_{-r_{0}}\left\{T_{r_{0}}, \sqrt{|p|}\right\}}{\mathrm{i} 8 \pi \beta G r_{0}} .
$$

which holds for every $r_{0} \neq 0$. Note that $\sqrt{|p|} \propto V^{1 / 3}$ where $V$ is the volume ${ }^{15}$ so that (2.20) is indeed analogous to (2.14). Note that the spectrum of the volume operator is essentially given by $|r|^{3 / 2}$ with $T_{r}$ as normalizable eigenfunctions (it takes a continuous range but consists only of eigenvalues, there is no continuous part). Hence the only zero-volume state is $T_{0}=1$. It follows immediately that if the inverse scale factor can be densely defined on the $T_{s}$ then at zero volume it is bounded.

Expression (2.20) is readily quantized by substituting Poisson brackets by commutators divided by $\mathrm{i} \hbar$. This gives

\footnotetext{
${ }^{14}$ What follows is a drastically simplified version of [37].

${ }^{15}$ For $k=0$ this volume is actually infinite. We mean here the volume divided by the comoving volume.
} 
$\frac{\widehat{1}}{\sqrt{|p|}} T_{r} \propto \frac{T_{r_{0}} \sqrt{|p|} T_{-r_{0}}-T_{-r_{0}} \widehat{\sqrt{|p|}} T_{r_{0}}}{\beta \ell_{P}^{2} r_{0}} T_{r}=\frac{\sqrt{\left|r+r_{0}\right|}-\sqrt{\left|r-r_{0}\right|}}{r_{0} \sqrt{\beta} \ell_{P}} T_{r}$,

which is bounded (in fact vanishes) at $r=0$. It is even bounded for all $r$ and coincides with the operator $1 / \sqrt{|\hat{p}|}$ for $r \gg r_{0}$. The parameter $r_{0}$ is a quantization ambiguity, called a factor ordering ambiguity which is also present in LQG [38] although it is there labelled by a discrete rather than continuous parameter. Note that by the constraint $C=C_{\text {geo }}+C_{\text {scalar }}=0$ we obtain $q^{2} \propto p^{2} /\left(2 a^{4}\right)+V a^{2}$ which means that $q \propto a^{-2}$ at $a \rightarrow 0$ (see the discussion at the end of section 4) so that $C_{\text {geo }} \propto a^{-3}$ classically. However, quantum mechanically $C_{\text {geo }}$ is bounded at $a \rightarrow 0$ because $q$ is quantized by the bounded expression $\left(T_{r_{0}}-T_{-r_{0}}\right) /\left(2 \mathrm{i} r_{0}\right)$.

So far we have only discussed the isotropic model. In the non-isotropic but diagonal model [20] similar remarks apply because the three scale factors mutually commute. Hence the inverse scale factors can be treated separately just as in the isotropic case.

Let us summarize: that LQC is the usual cosmological minisuperspace phase space quantized by LQG methods. It borrows in an essential way the Hilbert space [8] and the key technique [13]. LQC therefore seems to confirm the aspects [8, 13] for LQG.

However, despite these similarities there are important differences:

To begin with, LQC does not have a graph label. That is of course expected because in cosmology all points are equivalent due to homogeneity, hence there is only one point and thus no room for a graph. Consequently, there is no such thing as the valence of a vertex. These facts lead to an enormous simplification of the volume spectrum which is only modestly degenerate (multiplicity two for all $r \neq 0$, multiplicity one for $r=0$ ) while in LQG the volume spectrum is infinitely degenerate, in particular for zero eigenvalues (just apply a diffeomorphism to a given volume eigenstate which changes the graph). Moreover, the volume spectrum in LQG has discrete range while in LQC it has continuous range. Next, in LQG the Hamiltonian constraint modifies the graph of a spin network state while in LQC that is not possible since there is no graph. That leads to an enormous simplification concerning the computation of the kernel of the Hamiltonian constraint which boils down to the solution of a recursion relation within a finite-dimensional parameter space while in LQG the corresponding parameter space [15] is infinite dimensional. Finally, while it is possible to interpret the LQC states as distributional LQG states [39] (simply multiply an ordinary LQG state by a $\delta$ distribution supported on homogeneous connections) this distributional space of states is not preserved by the (dual) action of the operators of LQG, hence LQC is not an invariant (distributional) sector of LQG. It really is a model whose connection to LQG involves the classical truncation, thus inhomogeneous fluctuations are switched off by hand rather than being quantum mechanically suppressed.

We conclude that by definition of a toy model with a finite-dimensional phase space, LQC cannot model the QFT aspects of LQG. LQC models very successfully two aspects of LQG, namely those of $[8,13]$. However, it is far from granted that those predictions of LQC which are sensitive to the QFT aspects will hold in LQG as well. As we just saw, the spectrum of the inverse scale factor is sensitive to those aspects since the volume operator is. It is precisely one of the purposes of the present paper to investigate whether these differences lead to a qualitatively different picture in LQG when applied to cosmology.

\section{Unboundedness of the analogon of the inverse scale factor in full LQG}

We can explain the reason for the unboundedness of the operator concerning the inverse scale factor in very intuitive terms without going through the (admittedly lengthy) analysis. We will compare this to the isotropic LQC model, similar arguments apply to the diagonal 
models: the spectrum of the inverse scale factor can, roughly speaking, be obtained by taking the difference between neighbouring eigenvalues of the volume operator (discrete derivative). This is the case because, according to (2.14), the inverse scale factor can be obtained from the Ko-Dreibein as $e=\{A, V\}$ where $V$ is the volume and $A$ is the connection. This is the key technique of [13] as mentioned above. Upon quantization the connection gets replaced by a holonomy, that is, $\hat{e} \propto h\left[h^{-1}, \hat{V}\right]=V-h \hat{V} h^{-1}$ where $h$ is the holonomy of $A$ along an edge. The holonomy changes the spin labels of a spin network state by one unit, hence we get a discrete derivative in the spin parameter space. Now the spectrum of the volume operator depends parametrically, among other things, precisely on the spins of the edges of a spin network attached to a vertex and additional intertwiner quantum numbers and this is the discrete derivative of the volume spectrum that we mentioned.

If we view the spectrum of the volume operator as a function of these parameters then in isotropic LQC this parameter space is one dimensional, it is a function $j \mapsto V(j)$ (in LQC the parameter $j:=r$ of the previous section takes continuous and not discrete values but that does not change the qualitative picture). In LQG on the other hand it is arbitrarily high dimensional, the dimensionality being governed by the number $n$ of vertices and the valences $N_{v}$ of the individual vertices, it is a function of the form $\left(j_{1}^{(1)}, \ldots, j_{N_{n}}^{(n)}\right) \mapsto \sum_{v=1}^{n} V_{v}\left(j_{1}^{(v)}, \ldots, j_{N_{v}}^{(v)}\right)$ where the $n, N_{v}$ can be arbitrarily large. In both cases the functions $V$ are nowhere singular except when at least one of the $j$ tends to infinity.

The point is now that in isotropic LQC the zero-volume condition $V(j)=0$ has a finite number of solutions $j=j_{0}<\infty$ (in fact one). The spectrum of the inverse scale factor at zero volume is then, roughly speaking, given by $V\left(j_{0}+\delta j\right)-V\left(j_{0}\right)$ (using suitable discrete units for the parameters $j$, in LQC $\delta j=r_{0}$ ) which is finite since $j_{0}<\infty$ whence we get boundedness at zero volume. In LQG on the other hand the situation is more interesting: the zero-volume condition now gives an infinite number (parameter space of codimension one) of solutions. Pictorially speaking, the spectrum of the volume operator plotted against the parameter space can be envisioned as a complicated landscape of mountains which is disrupted by many valleys of zero volume which intersect each other in a complicated way. The boundaries of those valleys have arbitrarily steep inclination and the mountains are arbitrarily high. This is because the solutions of the zero-volume condition are now of the form $j_{1}^{(v)}=j_{0}^{(v)}\left(j_{2}^{(v)}, \ldots, j_{N_{v}}^{(v)}\right)$ and we may let $j_{2}^{(v)}, \ldots, j_{N_{v}}^{(v)} \rightarrow \infty$ in many ways (along many valleys). It is clear that now for each $v V_{v}\left(\left(j_{1}^{(v)}=j_{0}^{(v)}, j_{2}^{(v)}, \ldots, j_{N_{v}}^{(v)}\right)\right)-V_{v}\left(\left(j_{1}^{(v)}=j_{0}, j_{2}^{(v)}, \ldots, j_{N_{v}}^{(v)}\right)+\delta j^{(v)}\right)$ with $\delta j_{k}^{(v)}=\delta_{k l}, l \in\left\{1, \ldots, N_{v}\right\}$ can become arbitrarily large. This is what we are going to prove explicitly in our companion paper [28] based on the recent simplification of the analytical expression for the matrix elements of the volume operator [35] ${ }^{16}$.

One may object that the diverging configurations $\left(j_{1}^{(v)}, j_{2}^{(v)}, \ldots, j_{N_{v}}^{(v)}\right)$ for each individual $v$ do not look isotropic in general and that one should require isotropy as in LQC which perhaps should enforce $j_{1}^{(v)}=\cdots=j_{N_{v}}^{(v)}$ which could lead to a bound since the parameter space is now one dimensional ${ }^{17}$. However, here we now invoke a second key difference between LQC and LQG: LQC is a quantum-mechanical toy model of LQG. It can be thought of as LQG where all the vertices of a spin network state are to be thought of as equivalent due to homogeneity. In other words, LQC is like LQG except that we only consider spin network states with a single vertex (and a few edges, usually no more than three). LQG on the other hand is a field theory and spin network states can have an arbitrary number of vertices. Consider now a zero-volume spin network state $T_{\lambda, v}$ where $\lambda$ is the eigenvalue of the inverse scale factor and $v$ is a vertex at

\footnotetext{
${ }^{16}$ For the very special case of matrix elements we are going to use a similar expression that was also derived in [36] using a different method.

${ }^{17}$ Indeed, if we choose $j_{1}=j_{2}=j_{3}=j$ in formula (3.5) then the result is bounded in $j$.
} 


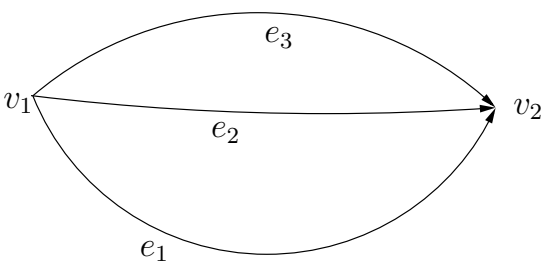

Figure 1. Simplest three valent gauge invariant graph $\gamma: V(\gamma)=\left\{v_{1}, v_{2}\right\}, E(\gamma)=\left\{e_{1}, e_{2}, e_{3}\right\}$.

which the graph $\gamma_{v}$ with a single non-coplanar ${ }^{18}$ vertex $v$ of the spin network state is located. Let $S$ be a finite ${ }^{19}$ set of $n$ vertices obtained from a random sprinkling of points into the spatial manifold [40] and consider the state $\psi:=\otimes_{v \in S} T_{\lambda, v}$. We may arrange that the $\gamma_{v}$ are mutually disjoint and that their orientation is random as well. This state is homogeneous and isotropic on large scales but not on microscopic ones (the violation scale of homogeneity and isotropy depends on the number of points in $S$ and decreases with $n$ ). The state is normalized and still an eigenvalue $\lambda$ eigenstate of the inverse scale factor. This proves that we may construct kinematical states with large scale homogeneity and isotropy in LQG with arbitrarily large norm of the inverse scale factor ${ }^{20}$.

Let us now go into more details: in order to match the LQC calculations as closely as possible, what we really have studied is the contribution of a scalar (or inflaton) field to the Hamiltonian constraint (smeared energy density) operator whose classical expression is

$$
2 C_{\text {scalar }}(N)=\int_{\sigma} \mathrm{d}^{3} x N\left[\frac{\pi^{2}}{\sqrt{\operatorname{det}(q)}}+\sqrt{\operatorname{det}(q)}\left(q^{a b} \phi_{, a} \phi_{, b}+2 V(\phi)\right)\right] .
$$

Here $\phi$ is the scalar field, $\pi$ its conjugate momentum, $q_{a b}$ is the pull back to the spatial manifold $\sigma$ of the four metric $g_{\mu \nu}$ on the four manifold $M \cong \mathbb{R} \times \sigma$ and $N$ is a test function (see also section 2). In an exactly homogeneous situation, say the FRW metric, the derivative term drops out and the potential term becomes proportional to $a^{3}$ while the kinetic term becomes proportional to $a^{-3}$ where $a$ is the scale factor of the FRW metric. The classical initial singularity $\lim _{t \rightarrow 0} a(t)=0$ is therefore prominent in the kinetic term. Of course in full LQG the kinetic term must be and can be considered as well; see [15]. But it will be sufficient to consider the kinetic term to demonstrate our point.

Consider therefore the kinetic term

$$
2 C_{\text {scalar }}^{\text {kin }}(N)=\int_{\sigma} \mathrm{d}^{3} x N \frac{\pi^{2}}{\sqrt{\operatorname{det}(q)}} .
$$

Its quantization is derived in [15] on the kinematical Hilbert space $\mathcal{H}_{\text {kin }}=\mathcal{H}_{\text {geo }} \otimes \mathcal{H}_{\text {scalar }}$. An orthonormal basis in this Hilbert space [42] consists of the charge-spin network states $T_{\gamma, \vec{j}, \vec{I}} \otimes T_{V(\gamma), \vec{n}}$. Here $\gamma$ is a graph with vertex set $V(\gamma), \vec{j}$ denotes a labelling of each edge $e$ of $\gamma$ by half integral spin quantum numbers $j_{e}$, and $\vec{I}, \vec{n}$ respectively denote a labelling of each vertex $v$ of $\gamma$ with a gauge invariant intertwiner $I_{v}$ and integer $n_{v}$ respectively. The action of the corresponding operator, densely defined in the finite linear span of this basis, can now schematically be written as

\footnotetext{
18 The volume operator vanishes on (non) gauge invariant vertices which are not at least (tri) four valent and at which not at least three tangents of the adjacent edges are linearly independent.

19 This will be sufficient if the spatial manifold is compact as for $k=1$ FRW models or if we are interested only a compact, observationally accessible (inside the Hubble radius) region. In the $k=0,-1$ case we can use in the infinite tensor product [41].

${ }^{20}$ This is a purely kinematical result as in LQC. In fact it must be because the inverse scale factor itself is not a Dirac
} observable, see the next section. 
$\widehat{2 C_{\text {scalar }}^{\text {kin }}}(N) T_{\gamma, \vec{j}, \vec{I}, \vec{n}}=\sum_{v \in V(\gamma)} N(v)\left[\frac{\widehat{1}}{\sqrt{\operatorname{det}(q)}}(v) T_{\gamma, \vec{j}, \vec{I}}\right] \otimes\left[\widehat{\pi}(v)^{2} T_{V(\gamma), \vec{n}}\right]$.

The explicit meaning and action of the genuine operators (not operator valued distributions) can be found in $[15,34]$ and is also reviewed in [28] for the convenience of the reader. For the purpose of this paper it is enough to report the result of our calculation.

The analogon of the LQC inverse scale factor (cubed) in LQG is of course the non-negative

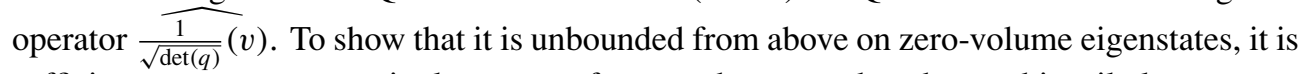
sufficient to construct one single system of states whose members have arbitrarily large norm. The simplest zero-volume eigenstates are spin network states $T_{\gamma, \vec{j}, \vec{I}}$ which have at most three valent vertices. They are very simple because the intertwiners $I_{v}$ are then uniquely determined. We are thus interested in the numbers

$$
\left\|\frac{\widehat{1}}{\sqrt{\operatorname{det}(q)}}(v) T_{\gamma, \vec{j}}\right\|^{2}=\left\langle T_{\gamma, \vec{j}},\left[\frac{\widehat{1}}{\sqrt{\operatorname{det}(q)}}(v)\right]^{2} T_{\gamma, \vec{j}}\right\rangle,
$$

where $v$ is a three valent vertex of $\gamma$ whose adjacent, non-coplanar edges carry the spin quantum numbers $j_{1} \leqslant j_{2} \leqslant j_{3}$ subject to the constraint that $j_{3} \in\left\{j_{1}+j_{2}, j_{1}+j_{2}-1, \ldots,\left|j_{1}-j_{2}\right|\right\}$. To make things definite, consider a graph $\gamma$ with three edges and two three valent, non-coplanar vertices; see figure 3 .

Remarkably the number (3.4) can be computed analytically. This is possible due to a recent improvement in the technology for the computation of the matrix elements of the volume operator for full LQG [35]. The analytical formula for (3.4) is given by the surprisingly compact expression

$$
\begin{aligned}
&\left\|\widehat{\frac{1}{\sqrt{\operatorname{det}(q)}}(v)} T_{\gamma, \vec{j}}\right\|^{2}=\frac{C^{2}}{\left(\ell_{P}\right)^{6}} \cdot\left[\frac { 3 2 } { 9 ( 1 + 2 j _ { 1 } ) ^ { 2 } ( 1 + 2 j _ { 2 } ) ^ { 2 } ( 1 + 2 j _ { 3 } ) ^ { 2 } } \left[108 A_{1} A_{2} A_{3}-3\left(2(-1)^{2 j_{1}}\right.\right.\right. \\
&\left.+(-1)^{2 j_{3}}\right)^{2} A_{2}\left(A_{1}-A_{2}+A_{3}\right)^{2}-3\left(2(-1)^{2 j_{2}}+(-1)^{2 j_{3}}\right)^{2} A_{1}\left(-A_{1}+A_{2}+A_{3}\right)^{2} \\
&-3\left(1+2(-1)^{2\left(j_{1}+j_{2}\right)}\right)^{2} A_{3}\left(A_{1}+A_{2}-A_{3}\right)^{2}-\left(1+2(-1)^{2\left(j_{1}+j_{2}\right)}\right) \\
& \times\left(2(-1)^{2 j_{1}}+(-1)^{2 j_{3}}\right)\left(2(-1)^{2 j_{2}}+(-1)^{2 j_{3}}\right)\left(-A_{1}+A_{2}+A_{3}\right)\left(A_{1}-A_{2}+A_{3}\right) \\
&\left.\left.\times\left(A_{1}+A_{2}-A_{3}\right)\right]\left(V_{1 A^{\frac{1}{4}}}-V_{1 B^{\frac{1}{4}}}\right)^{2}\left(V_{2 A^{\frac{1}{4}}}-V_{2 B^{\frac{1}{4}}}\right)^{2}\left(V_{3 A^{\frac{1}{4}}}-V_{3 B^{\frac{1}{4}}}\right)^{2}\right]^{2}
\end{aligned}
$$

where $C$ is a positive numerical constant depending on the regularization of the operator $\frac{\widehat{1}}{\sqrt{\operatorname{det}(q)}}(v)$ and the volume operator. Furthermore $\left(j_{1}, j_{2}, j_{3}\right)$ are the spins of the three edges $\left(e_{1}, e_{2}, e_{3}\right)$ outgoing at the vertex $v$ and $A_{K}:=j_{K}\left(j_{K}+1\right)$. Moreover

$$
\begin{aligned}
V_{1 A} & =\left[\left(-j_{1}+j_{2}+j_{3}+1\right)\left(j_{1}-j_{2}+j_{3}\right)\left(j_{1}+j_{2}-j_{3}\right)\left(j_{1}+j_{2}+j_{3}+1\right)\right]^{\frac{1}{2}} \\
V_{1 B} & =\left[\left(-j_{1}+j_{2}+j_{3}\right)\left(j_{1}-j_{2}+j_{3}+1\right)\left(j_{1}+j_{2}-j_{3}+1\right)\left(j_{1}+j_{2}+j_{3}+2\right)\right]^{\frac{1}{2}} \\
V_{2 A} & =\left[\left(-j_{1}+j_{2}+j_{3}\right)\left(j_{1}-j_{2}+j_{3}+1\right)\left(j_{1}+j_{2}-j_{3}\right)\left(j_{1}+j_{2}+j_{3}+1\right)\right]^{\frac{1}{2}} \\
V_{2 B} & =\left[\left(-j_{1}+j_{2}+j_{3}\right)\left(j_{1}-j_{2}+j_{3}\right)\left(j_{1}+j_{2}-j_{3}+1\right)\left(j_{1}+j_{2}+j_{3}+2\right)\right]^{\frac{1}{2}} \\
V_{3 A} & =\left[\left(-j_{1}+j_{2}+j_{3}\right)\left(j_{1}-j_{2}+j_{3}\right)\left(j_{1}+j_{2}-j_{3}+1\right)\left(j_{1}+j_{2}+j_{3}+1\right)\right]^{\frac{1}{2}} \\
V_{3 B} & =\left[\left(-j_{1}+j_{2}+j_{3}+1\right)\left(j_{1}-j_{2}+j_{3}+1\right)\left(j_{1}+j_{2}-j_{3}\right)\left(j_{1}+j_{2}+j_{3}+2\right)\right]^{\frac{1}{2}} .
\end{aligned}
$$




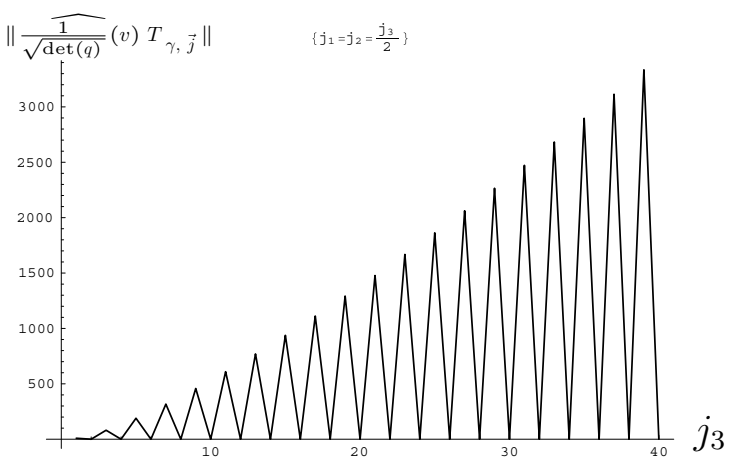

Figure 2. Plot for $j_{1}=j_{2}=\frac{j_{3}}{2}$ where $j_{3} \in \mathbb{N}$ with $1 \leqslant j_{3} \leqslant 40$. The graph oscillates between 0 (if $j_{3}$ even) and an increasing value (if $j_{3}$ odd).

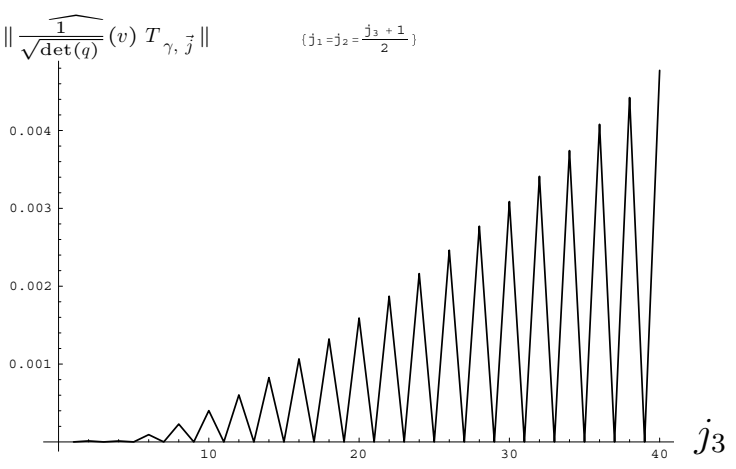

Figure 3. Plot for $j_{1}=j_{2}=\frac{j_{3}+1}{2}$ where $j_{3} \in \mathbb{N}$ with $1 \leqslant j_{3} \leqslant 40$. The graph oscillates between 0 (if $j_{3}$ odd) and an increasing value (if $j_{3}$ even).

We now display a few diverging series of configurations $\left(j_{1}, j_{2}, j_{3}\right)$ where we always plot $\left\|\frac{1}{\sqrt{\operatorname{det}(q)}}(v) T_{\gamma, \vec{j}}\right\|:=\sqrt{\| \frac{1}{\sqrt{\operatorname{det}(q)}}(v)} T_{\gamma, \vec{j}} \|^{2}$, neglecting the prefactor $\frac{C}{\left.\left(\ell_{P}\right)^{3}\right)}$

Oscillating: $j_{1}=j_{2}=\frac{j_{3}}{2}$. If we set $j_{1}=j_{2}=\frac{j_{3}}{2}$ where $j_{3} \in \mathbb{N}$, we get

$\left\|\frac{\widehat{1}}{\sqrt{\operatorname{det}(q)}}(v) T_{\gamma, \vec{j}}\right\| \propto \frac{128 \sqrt{2}\left(-1+(-1)^{j_{3}}\right) j_{3}{ }^{4}\left(-3\left(2+(-1)^{j_{3}}\right)+\left(1+(-1)^{3 j_{3}}\right) j_{3}\right)}{9\left(1+j_{3}\right)\left(1+2 j_{3}\right)^{\frac{7}{4}}}$.

Asymptotically this increases as

$$
\begin{aligned}
& \left\|\frac{\widehat{1}}{\sqrt{\operatorname{det}(q)}}(v) T_{\gamma, \vec{j}}\right\| \propto \frac{128 \cdot 6 \cdot \sqrt{2} \cdot j_{3}{ }^{4}}{9\left(1+j_{3}\right)\left(1+2 j_{3}\right)^{\frac{11}{4}}} \\
& \stackrel{j_{3} \rightarrow \infty}{\propto} 35.9 \cdot j_{3}{ }^{\frac{5}{4}}
\end{aligned}
$$

$j_{1}=j_{2}=\frac{j_{3}}{2}+\frac{1}{2}$. If we set $j_{1}=j_{2}=\frac{j_{3}}{2}+\frac{1}{2}$ where $j_{3} \in \mathbb{N}$, we get

$$
\begin{aligned}
\| \frac{\widehat{1}}{\sqrt{\operatorname{det}(q)}}(v) & T_{\gamma, \vec{j}} \| \propto \frac{1}{9\left(2+j_{3}\right)^{4}\left(1+2 j_{3}\right)^{2}} 128 \sqrt{2}\left(1+(-1)^{j_{3}}\right) j_{3}{ }^{2}\left(1+j_{3}\right)^{3} \\
& \times\left(-3\left(-7+3(-1)^{j_{3}}+(-1)^{3 j_{3}}\right)+\left(-1+(-1)^{3 j_{3}}\right) j_{3}\right)\left(-\left(2^{\frac{1}{4}}\left(j_{3}{ }^{2}\left(1+j_{3}\right)\right)^{\frac{1}{8}}\right)\right. \\
& \left.+\left(\left(1+j_{3}\right)^{2}\left(3+2 j_{3}\right)\right)^{\frac{1}{8}}\right)^{2}\left(\left(j_{3}\left(1+j_{3}\right)^{2}\right)^{\frac{1}{8}}-\left(j_{3}\left(3+5 j_{3}+2 j_{3}{ }^{2}\right)\right)^{\frac{1}{8}}\right)^{4} .
\end{aligned}
$$


Asymptotically this increases as

$$
\left\|\frac{1}{\sqrt{\operatorname{det}(q)}}(v) T_{\gamma, \vec{j}}\right\| \propto 5.9 \times 10^{-5} j_{3}{ }^{\frac{5}{4}} .
$$

Increasing: $j_{1}=\frac{3}{2} j_{2}=j_{3}+\frac{1}{2}$. If we set $j_{1}=\frac{3}{2}, j_{2}=j_{3}+\frac{1}{2}$ where $j_{3} \in \mathbb{N}$, we get:

$$
\begin{aligned}
& \left\|\frac{\widehat{1}}{\sqrt{\operatorname{det}(q)}}(v) T_{\gamma, \vec{j}}\right\| \propto \frac{1}{3\left(1+j_{3}\right)\left(1+2 j_{3}\right)^{2}} 4 j_{3}\left(-9+21 j_{3}+14 j_{3}{ }^{2}\right)\left(-\left(3^{\frac{1}{8}}\left(j_{3}\left(2+j_{3}\right)\right)^{\frac{1}{8}}\right)\right. \\
& \left.+\left(-3+4 j_{3}+4 j_{3}{ }^{2}\right)^{\frac{1}{8}}\right)^{2}\left(-2\left(j_{3}\left(2+j_{3}\right)\right)^{\frac{1}{8}}+\sqrt{2} 3^{\frac{1}{8}}\left(-3+4 j_{3}+4 j_{3}{ }^{2}\right)^{\frac{1}{8}}\right)^{2} \\
& \times\left(\left(j_{3}\left(3+2 j_{3}\right)\right)^{\frac{1}{8}}-\left(-6+9 j_{3}+6 j_{3}^{2}\right)^{\frac{1}{8}}\right)^{2} \text {. }
\end{aligned}
$$

Asymptotically this increases as

$$
\left\|\frac{\widehat{1}}{\sqrt{\operatorname{det}(q)}}(v) T_{\gamma, \vec{j}}\right\| \propto 1.06 \times 10^{-6} j_{3}{ }^{\frac{3}{2}} .
$$

Identical 0: If we set $j_{1}=j_{2}=j_{3}$ and more general $j_{1}, j_{2}, j_{3} \in \mathbb{N}$ (all spins integer numbers) in (3.5) then

$$
\left\|\frac{\widehat{1}}{\sqrt{\operatorname{det}(q)}}(v) T_{\gamma, \vec{j}}\right\|=0 .
$$

General configurations. Finally we plot numbers (3.4) as $j_{1}, j_{2}$ vary over their allowed values at given $j_{3}$ for various values of $j_{3}$. The landscape of low valleys together with their arbitrarily high and steep boundaries is nicely illustrated: taking the general result (3.5) (without the prefactors $\left.\left(\ell_{P}\right)^{9}|Z|^{\frac{3}{2}}\right)$ we use the quantity

$Q= \begin{cases}30+\ln \left[\left\|\frac{\widehat{1}}{\sqrt{\operatorname{det}(q)}}(v) T_{\gamma, \vec{j}}\right\|\left(j_{1}, j_{2}, j_{3}\right)\right] & \left|j_{1}-j_{2}\right| \leqslant j_{3} \leqslant j_{1}+j_{2} \text { and } j_{1}+j_{2}+j_{3} \text { is an } \\ & \text { integer and }\left\|\frac{1}{\sqrt{\operatorname{det}(q)}}(v) T_{\gamma, \vec{j}}\right\|\left(j_{1}, j_{2}, j_{3}\right) \neq 0 \\ & \text { else }\end{cases}$

where ' 30 ' is arbitrarily added in order to shift the plot upwards. We make a three dimensional plot (in the range $\frac{1}{2} \leqslant j_{1} \leqslant j_{\max }, \frac{1}{2} \leqslant j_{2} \leqslant j_{\max }$ for each fixed value $5 \leqslant j_{3} \leqslant \frac{15}{2}$ : it turns out that the nonzero configurations are grouped symmetrically along lines parallel to the $j_{1}=j_{2}$ axis. The reason for this is of course the integer requirement $j_{1}+j_{2}+j_{3} \stackrel{!}{=}$ integer. Therefore we will get contributions on the $j_{1}=j_{2}$-axis only if $j_{3}$ is an integer. Because (3.5) is symmetric with respect to the interchange of $j_{1} \leftrightarrow j_{2}$ we may restrict ourselves to the range $j_{1} \geqslant j_{2}$. lines

Additionally we contribute for each of those 3D-plots a two-dimensional plot along the

$$
j_{2}=j_{1}-l \quad \text { with } 0 \leqslant l \leqslant \min \left[j_{3}, j_{\max }-\frac{1}{2}\right], \quad l+\frac{1}{2} \leqslant j_{1} \leqslant j_{\max }=25 .
$$

The restriction for the parameter $l$ is a result of the requirements $\left|j_{1}-j_{2}\right| \leqslant j_{3} \leqslant j_{1}+j_{2}$ from which for $j_{1}>j_{2}$ we may remove the modulus. In order to give a better impression we have joined only non-vanishing values of $Q$ along the lines described above $\mathrm{e}^{21}$.

\footnotetext{
${ }^{21}$ For the integer $j_{3}$ also every second configuration on the lines gives a 0 (the identically 0 case). Hence, if we were to join all the points, the plots would oscillate between 0 and the now plotted curves and it would be hardly possible to see anything useful from them.
} 


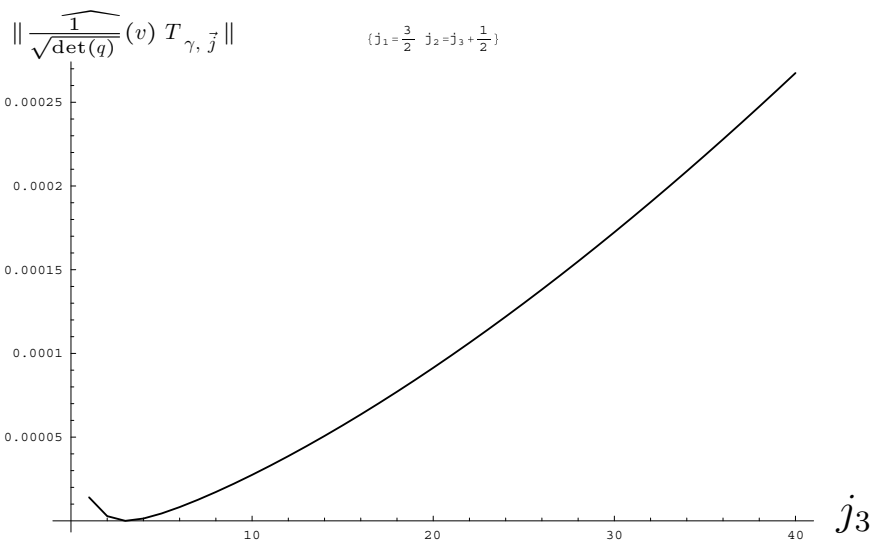

Figure 4. Plot for $j_{1}=\frac{3}{2}, j_{2}=j_{3}+\frac{1}{2}$ where $j_{3} \in \mathbb{N}$ with $1 \leqslant j_{3} \leqslant 40$. The graph first decreases for $1 \leqslant j_{3}<3$ and is 0 for $j_{3}=3$. It increases for $j_{3}>3$.

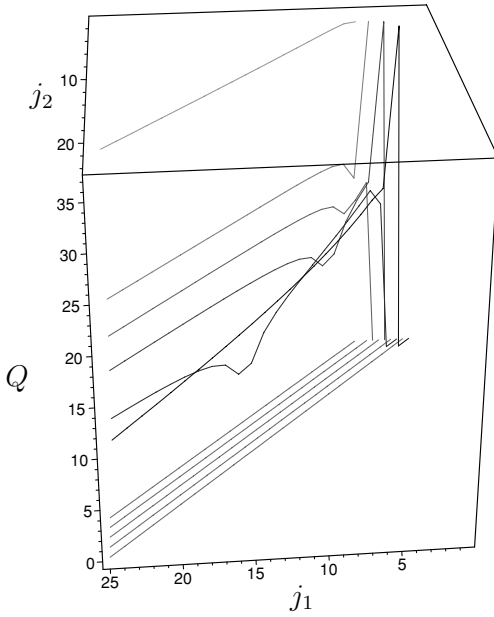

Figure 5. Plot for $j_{3}=5$.

Due to the large kernel of the volume operator even for four and higher valent vertices [35] qualitatively similar behaviour will prevail in those cases as well. The unboundedness of the 'inverse scale factor' operator in full LQG, even on homogeneous and isotropic states, is herewith established: let $\gamma_{1}, \ldots, \gamma_{n}$ be a random distribution of mutually disjoint but diffeomorphic graphs, say of the type of figure 3 which is homogeneous and isotropic with respect to the comoving spatial metric of a FRW spacetime on a scale large compared to the Planck length within a compact region (the smaller that scale the larger $n$ ). The combined graph $\gamma:=\cup_{k=1}^{n} \gamma_{k}$ then looks homogeneous and isotropic. Consider the state of unit norm

$$
T_{\gamma, \lambda}:=\otimes_{k=1}^{n} T_{\gamma_{k}, \vec{j}}
$$

where we choose the same triple of spins for every graph and such that the numbers (3.4) are equal to the same value $\lambda^{2}$ for every vertex of the $\gamma_{k}$. Then obviously all the norms (3.4) with $T_{\gamma, \vec{j}}$ chosen as the homogeneous state (3.8) equal $\lambda^{2}$ for every of the $2 n$ vertices of $\gamma$. But $\lambda$ can be arbitrarily large.

Let us compare this with the situation for the hydrogen atom: in both LQG and LQC the spectrum of the inverse scale factor is purely discrete, so we should compare with the bound 


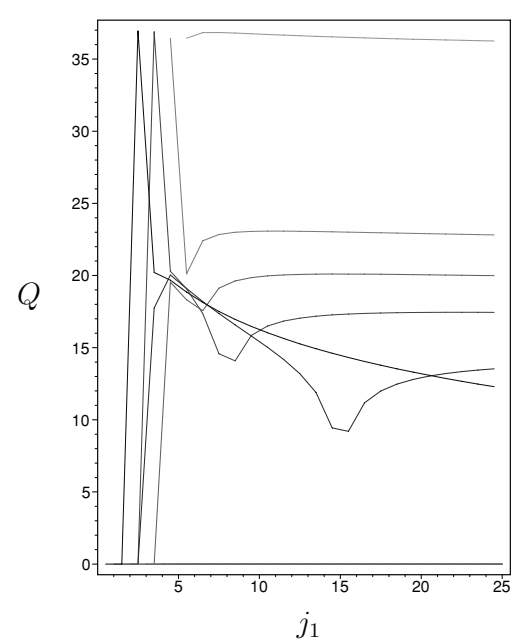

Figure 6. Plot for $j_{3}=5$.

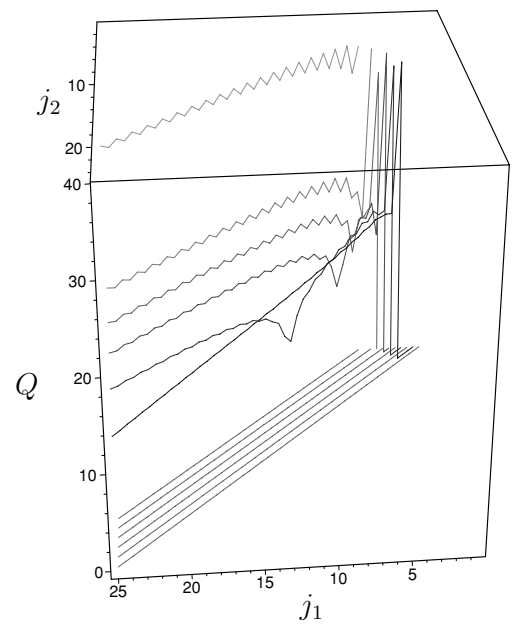

Figure 7. Plot for $j_{3}=\frac{11}{2}$.

states of the hydrogen atom. Restricted to the subspace of bound states it is indeed the case that the Hamiltonian of the hydrogen atom is bounded from below and above and this is not changed when we switch from the finite number of degrees of freedom of QM to the infinite number of degrees of freedom of QED. However, the interplay between LQC and LQG is different: in LQC we have boundedness, but after switching on LQG we get unboundedness.

\section{Boundedness of the inverse scale factor expectation values at the initial singularity}

The result of the previous section sounds rather negative at first. However, it is not as we will now explain. It just shows that the boundedness of the inverse scale factor cannot be a mechanism for avoidance of the local singularity in LQG.

Namely, what we have shown is unboundedness on certain homogeneous and isotropic spin network states. However, that does not mean that the inverse scale factor is unbounded on all homogeneous and isotropic states. In fact, we should worry only about boundedness on 


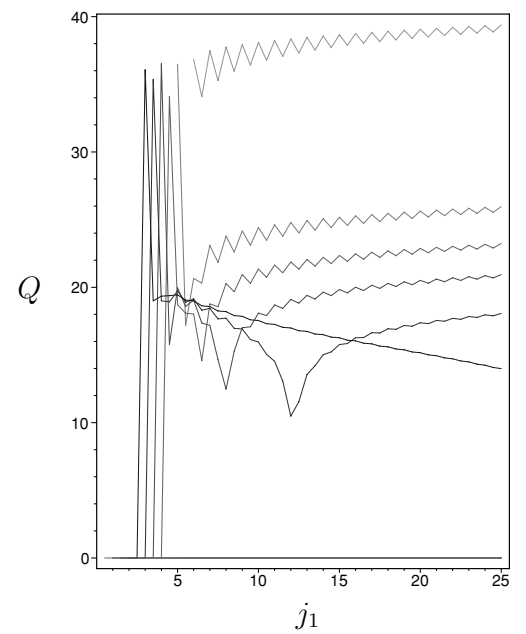

Figure 8. Plot for $j_{3}=\frac{11}{2}, j_{2}=j_{1}-l$. The different curves are (bottom to top): $l=\frac{1}{2}, \frac{3}{2}, \ldots, \frac{11}{2}$.

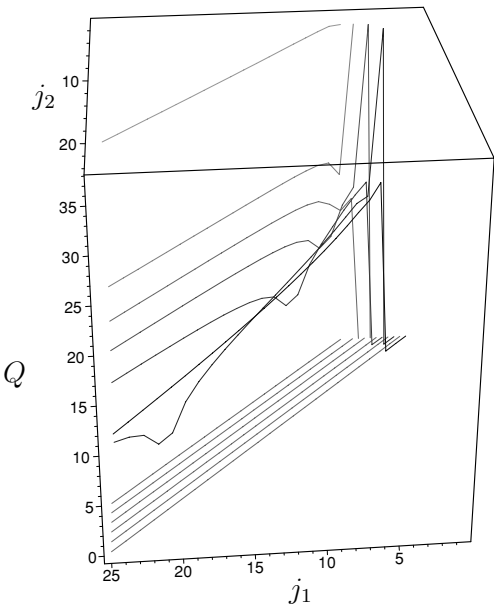

Figure 9. Plot for $j_{3}=6$.

those states which describe a collapsing universe which is homogeneous and isotropic on large scales. Indeed, in this section we will display the result of a calculation which indicates that the expectation value of the inverse scale factor with respect to (kinematical) coherent states [43] peaked on any homogeneous and isotropic point in phase space (e.g. a FRW universe along the classical singular trajectory) is bounded from above in $L Q G$ even at the big bang.

The details of our calculation can be read in the companion paper [28]. However, again we can explain the basic mechanism for this boundedness result in very intuitive terms: spin network states are, roughly speaking, eigenstates for the kinematical geometric operators such as the triad operator out of which the inverse scale factor is assembled. They are thus maximally sharp for half of the degrees of freedom (the electric fields) but maximally unsharp for the other half (the connections) which are encoded in the holonomy operators. Coherent states intermediate in between those two uncertainties and in fact minimize the Heisenberg uncertainty bound as far as conjugate canonical pairs are concerned. This is achieved by superimposing basis states such as spin network states with carefully chosen 


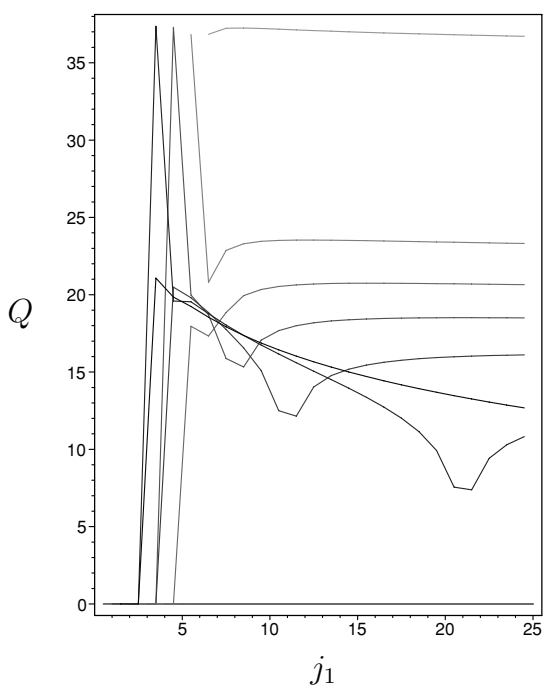

Figure 10. Plot for $j_{3}=6$.

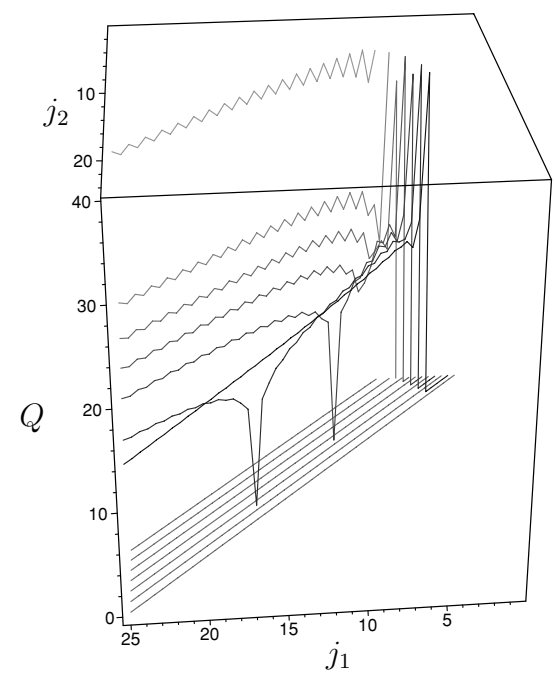

Figure 11. Plot for $j_{3}=\frac{13}{2}$.

coefficients. These coefficients depend on a point in the classical phase space, in our case a field configuration $(A, E)$ of connections and electric fields respectively. The coherent states are thus labelled by these points and they are peaked in phase space in the sense that the overlap function $\left|\left\langle\psi_{(A, E)}, \psi_{\left(A^{\prime}, E^{\prime}\right)}\right\rangle\right|^{2}$ vanishes exponentially fast unless $\left\|A-A^{\prime}\right\|^{2},\left\|E-E^{\prime}\right\|^{2} \leqslant s$ where $\|\cdot\|$ is a suitable norm on the classical field space and $s$ is a parameter which encodes the Gaussian width of the wave packet or the volume of a cell in phase space (it is proportional to $\hbar)$. The Gaussian decay of the overlap function implies that in terms of spin network states the state is of the form $\psi_{(A, E)}=\sum_{j} c_{j}(A, E) T_{j}$ where we have not displayed the graph label for simplicity and where the $c_{j}$ decay (symbolically) as $\exp \left(-s j^{2}\right)$. The label $j$ is a compound label for all participating spins and intertwiners. Now while the norm of the inverse scale factor in the states $T_{j}$ is unbounded from above that bound only diverges with a finite power 


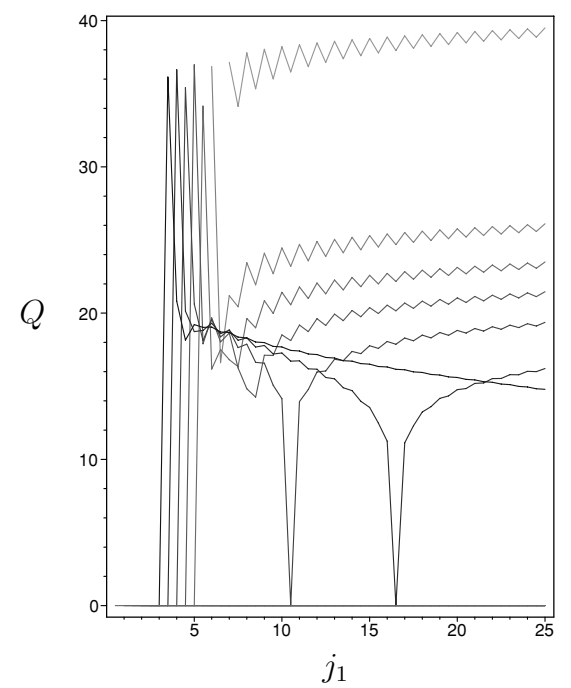

Figure 12. Plot for $j_{3}=\frac{13}{2}, j_{2}=j_{1}-l$. The different curves are (bottom to top): $l=\frac{1}{2}, \frac{3}{2}, \ldots, \frac{13}{2}$

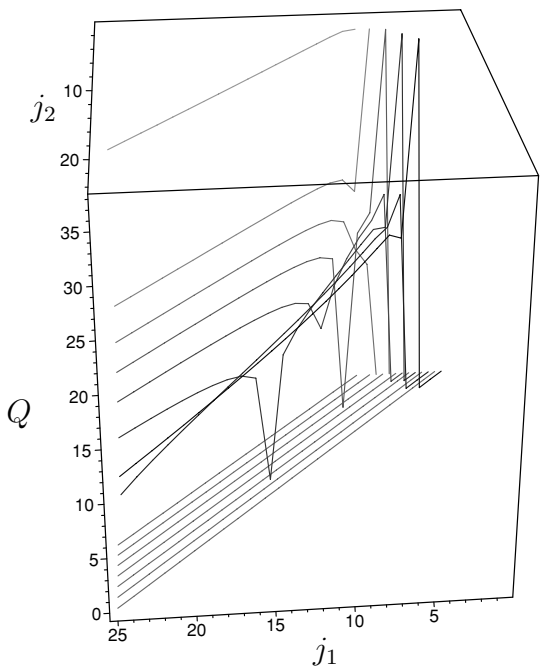

Figure 13. Plot for $j_{3}=7$.

as $j \rightarrow \infty$. This divergence therefore is completely swamped by the Gaussian decay of the exponential. This holds for rather generic values of $A, E$ and in particular when we choose a homogeneous and isotropic point in the phase space. Hence the mechanism is similar as in statistical physics: 'energy' (the argument of the Gaussian factor) wins over 'entropy' (the number of configurations leading to divergent eigenvalues). This a rather different mechanism which does not require boundedness of the inverse scale factor.

Let us go into more detail: given a point $\left(A_{0}, E_{0}\right)$ in the classical phase space, our (not normalized) coherent states are of the general form

$$
\psi_{\left(A_{0}, E_{0}\right)}=\sum_{\gamma} \psi_{\gamma,\left(A_{0}, E_{0}\right)}:=\left[e^{-\hat{\mathbf{C}} / \hbar} \delta_{A^{\prime}}\right]_{A^{\prime} \mapsto A^{\mathbb{C}}\left(A_{0}, E_{0}\right)},
$$




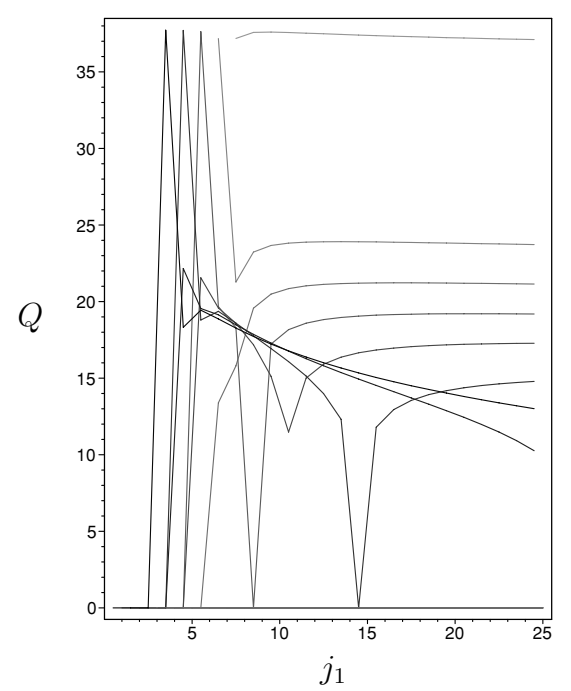

Figure 14. Plot for $j_{3}=7$.

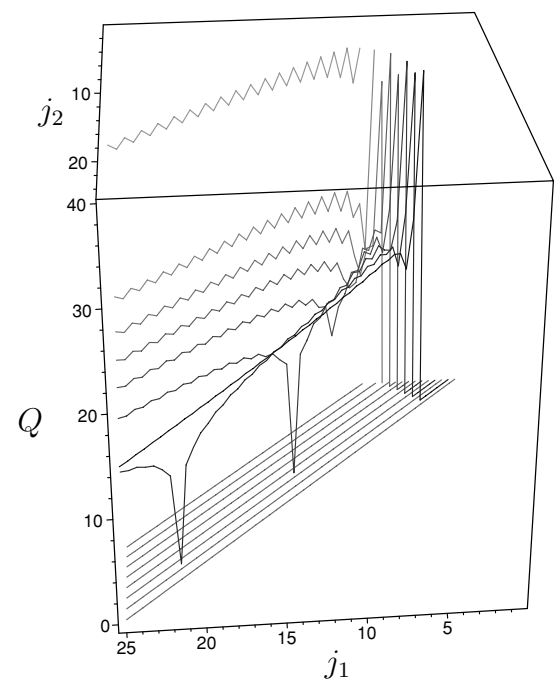

Figure 15. Plot for $j_{3}=\frac{15}{2}$.

where the uncountable sum extends over all finite graphs and $\psi_{\gamma,\left(A_{0}, E_{0}\right)}$ is a countably infinite coherent superposition of spin network states over $\gamma$. The coefficients in that superposition are uniquely determined by the generator $\mathbf{C}$ of the coherent states, also called the complexifier [47], which is supposed to have the dimension of an action. In fact, as we can see from (4.1), the coherent states are nothing else than the heat kernel (with respect to the operator C) evolution of the $\delta$ distribution with support at $A^{\prime}$ which is then analytically extended to $A^{\mathbb{C}}\left(A_{0}, E_{0}\right)$. The complexified connection $A^{\mathbb{C}}$ also is generated by $\mathbf{C}$ via

$A^{\mathbb{C}}:=\sum_{n=0}^{\infty} \frac{\mathrm{i}^{n}}{n !}\{\mathbf{C}, A\}_{(n)}=A+\mathrm{i}\{\mathbf{C}, A\}-\frac{1}{2}\{\mathbf{C},\{\mathbf{C}, A\}\}-\frac{\mathrm{i}}{6}\{\mathbf{C},\{\mathbf{C},\{\mathbf{C}, A\}\}\}+\cdots$.

This may look unfamiliar, however, this is precisely the way that the unnormalized coherent states for the harmonic oscillator are constructed for which the complexifier reads 
$\mathbf{C}=p^{2} /(2 m \omega)$ and the classicality parameter is $s=\hbar /(m \omega)$ (the role of the connection is of course played by the configuration coordinate $q$ ).

Ideally, according to the subsequent section, $\mathbf{C}$ should be adapted to a given Hamiltonian, but as we said, here we will carry out a completely kinematical calculation to get a first insight. It is therefore appropriate to choose $\mathbf{C}$ in such a way as to obtain the simplest possible states. One such choice involves the square of the area operator [47] which in turn is proportional to $E^{2}$ and thus will give rise to states very similar to those for the harmonic oscillator because in both cases the complexifiers are of the momentum squared type giving rise to Gaussian wave packets. Rather than defining $\mathbf{C}$ we directly display the corresponding states which in that case are, roughly, of the direct product form

$$
\psi_{\gamma,\left(A_{0}, E_{0}\right)}=\prod_{e \in E(\gamma)} \psi_{e,(A, E)}
$$

where

$$
\psi_{e,\left(A_{0}, E_{0}\right)}(A)=\sum_{2 j=1}^{\infty}(2 j+1) \mathrm{e}^{-\frac{t(e)}{2} j(j+1)} \operatorname{Tr}\left(\pi_{j}\left(g_{e}(A, E) A(e)^{-1}\right)\right) .
$$

Here $\pi_{j}$ is the spin $j$ irreducible representation of $S U(2)$. Note that the wavefunction $\psi_{e,\left(A_{0}, E_{0}\right)}(A)$ is a function of $A$ (or of the $S U(2)$ valued holonomies $A(e)$ ) which depends parametrically on $\left(A_{0}, E_{0}\right)$. The dimensionless parameter $t(e)$ depends on the details of $\mathbf{C}$ and satisfies $t(e)>0, t\left(e \circ e^{\prime}\right)=t(e)+t\left(e^{\prime}\right), t\left(e^{-1}\right)=t(e)$. Finally, $g_{e}\left(A_{0}, E_{0}\right) \approx$ $\exp \left(i \tau_{j} E_{0}^{j}\left(S_{e}\right) / 2\right) A_{0}(e)$ where $S_{e}$ is, roughly speaking, the face of a cell complex dual to $\gamma$ (i.e. each $S_{e}$ intersects $\gamma$ only in an interior point of $e$ ) which also depends on the details of C). Experts will see the representation theory of $S U(2)$ and the Peter-Weyl theorem at work in (4.3).

As explicitly shown in [43], expectation value computations with coherent states are qualitatively unaffected when we replace the group $S U(2)$ by $U(1)^{3}$ for which however the analysis simplifies tremendously. The corresponding coherent states are then given by

$$
\psi_{e,\left(A_{0}, E_{0}\right)}(A)=\sum_{\left|n_{1}\right|,\left|n_{2}\right|,\left|n_{3}\right|=1}^{\infty} \mathrm{e}^{-\frac{t(e)}{2} \sum_{j=1}^{3} n_{j}^{2}} \prod_{j=1}^{3}\left(g_{e}^{j}(A, E) A^{j}(e)^{-1}\right)^{n_{j}},
$$

where $A^{j}(e)=\exp \left(\mathrm{i} \int_{e} A^{j}\right)$ and where

$$
g_{e}^{j}\left(A_{0}, E_{0}\right)=\exp \left(\int_{S_{e}} \mathrm{~d} S_{a} E_{0 j}^{a}+\mathrm{i} \int_{e} A_{0}^{j}\right) .
$$

To carry out concrete calculations we just have to insert concrete values for $A_{0}, E_{0}$ into those formulae. In cosmological applications we choose, as in LQC (see section 2), e.g. $A_{0 a}^{j}=q \delta_{a}^{j}$ and $E_{0 j}^{a}=p \delta_{a}^{j}$ where $a=\sqrt{|p|}$ is the scale factor. Thus in LQG, rather than performing a cancellation of the inhomogeneous degrees of freedom as in LQC, we perform a suppression of their fluctuations by using appropriate states.

What one actually does in the computations is not to use the states $\psi_{\left(A_{0}, E_{0}\right)}$ but rather the 'cut-off' states $\psi_{\gamma,\left(A_{0}, E_{0}\right)}$ for finite but sufficiently fine $\gamma$. Roughly speaking, the analysis of $[43,47]$ shows that the optimal graph, for given resolution scale $L$ of a measurement ${ }^{22}$ should have an average edge length scale (with respect to the metric to be approximated) of the order of a geometric mean $\epsilon \approx L^{k} \ell_{P}^{1-k}$ where $0<k<1$ is a parameter that depends on the (partial) observables to be approximated. The reason for working with the cut-off states is

${ }^{22}$ Given a metric $g_{0}$ to be approximated, we want fluctuations in the length to be much smaller than $L$ where $L$ is to be measured with respect to $g_{0}$. On the other hand, the Planck scale discreteness of LQG forces $\epsilon \gg L$. 


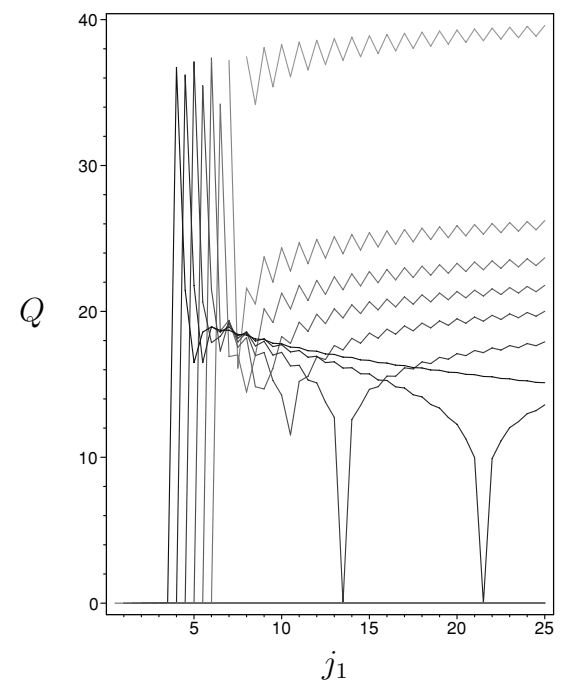

Figure 16. Plot for $j_{3}=\frac{15}{2}, j_{2}=j_{1}-l$. The different curves are (bottom to top): $l=\frac{1}{2}, \frac{3}{2}, \ldots, \frac{15}{2}$.

that the $\psi_{\left(A_{0}, E_{0}\right)}$ are not normalizable on the kinematical Hilbert space due to the sum over the uncountable number of graphs. They merely serve as a generator for the cut-off states which are normalizable.

What we have done then is to estimate from above the expectation value not only of the inverse scale factor in LQG for a general valence $M$ of the vertex for arbitrary $\left(A_{0}, E_{0}\right)$ but also the most general polynomials built out of generalized cotriad operators coming from classical expressions of the form $\left(\left\{A, V^{r}\right\}\right)^{N}$ which become upon quantization operators of the form $\left[\frac{\mathrm{i}}{\hbar \kappa}\right]^{N} \prod_{I=1}^{N} \hat{q}_{e_{I}}^{j}(v, r)=\left[\frac{\mathrm{i}}{\hbar \kappa}\right]^{N} \prod_{I=1}^{N} \hat{h}_{e_{I}}^{j}\left[\left(\hat{h}_{e_{I}}^{j}\right)^{-1}, \hat{V}^{r}\right]$ where $r, N$ are fixed, positive, rational and natural numbers respectively which are determined by the concrete coupling between matter and gravity [15]. Here $\hat{h}_{e}^{j}$ are the holonomies of the connection $A$ along edges of the graph, the classical volume expression $V$ turns into the volume operator $\hat{V}$ and Poisson brackets into commutators. A bound is given by ${ }^{23}$

$$
\begin{aligned}
\left\langle\Psi_{m, \gamma}^{(v)}(A)\left|\prod_{k=1}^{N} \hat{q}_{I_{k}}^{j_{k}}(r)\right| \Psi_{m, \gamma}^{(v)}(A)\right\rangle \leqslant\left(\ell_{P}\right)^{3 r N}(9 M)^{N}|Z|^{\frac{r N}{2}}\left[\frac{2 \mathbb{A}}{T^{2}}\right]^{N} \\
\times\left[\left[\frac{3 M p}{4 T}\right]^{N}+\sum_{n=1}^{N} \frac{N !}{(N-n) ! n !}\left[\frac{3 M p}{4 T}\right]^{N-n} \prod_{l=1}^{n}\left[\frac{3 M+2(l-1)}{4}\right]\right]
\end{aligned}
$$

where $M$ denotes the valence of the vertex, $N$ the degree of the polynomial, $Z$ is a constant numerical prefactor, depending on the Immirzi parameter and the regularization of the volume operator.

Moreover

$$
T:=\min _{I}\left\{T_{I}\right\} \rightarrow \mathbb{A}:=1+\frac{p}{T} \quad p:=\max _{I, j}\left\{\left|p_{I}^{j}(m)\right|\right\}
$$

with $T_{I}=\sqrt{t\left(e_{I}\right)}, t\left(e_{I}\right)$ is the classicality parameter mentioned above and $p_{I}^{j}(m) \approx$ $\frac{\epsilon}{L^{3}} \int_{S_{e_{I}}} \mathrm{~d} S_{a} E_{j}^{a}$. Here $m=\left(A_{0}, E_{0}\right)=(A, E)$ is the point in classical phase space the coherent

\footnotetext{
${ }^{23}$ Neglecting all terms of order $t^{\infty}$.
} 
state is peaked on and $L, \epsilon$ respectively are the resolution scale and graph scale mentioned above.

Specializing to homogeneous data $m=\left(A_{0 a}^{j}, E_{0 j}^{a}\right)=\left(\tilde{q} \delta_{a}^{j}, \tilde{p} \delta_{j}^{a}\right)$ gives $p=\tilde{p}$. $\max _{I} C_{I}^{\text {(construct) }}$, with numerical constants $C_{I}^{\text {(construct) }}$ carrying information about the details of the coherent state.

Taking the limit $a \rightarrow 0$ which causes $p=0, \mathbb{A}=1$ demonstrates that nothing dramatic happens and we get the huge but finite bound

$\left.\left\langle\Psi_{m, \gamma}^{(v)}(A)\left|\prod_{k=1}^{N} \hat{q}_{I_{k}}^{j_{k}}(r)\right| \Psi_{m, \gamma}^{(v)}(A)\right\rangle\right|_{a=0} \leqslant\left(\ell_{P}\right)^{3 r N}(9 M)^{N}|Z|^{\frac{r N}{2}}\left[\frac{2}{T^{2}}\right]^{N} \prod_{l=1}^{N}\left[\frac{3 M+2(l-1)}{4}\right]$.

For the case of the inverse scale factor we have to set $(r=1 / 2, N=6)$ and to multiply by $\frac{1}{(\hbar \kappa)^{N}}=\frac{1}{\left(\ell_{P}\right)^{12}}$.

These results prove our claim: the expectation value (and thus also the norm, just double $N$ ) of the inverse scale factor is bounded with respect to kinematical semiclassical states even when the classical metric that labels the coherent state becomes maximally degenerate. As in LQC this is a strong indication for a drastic modification of the effective Friedmann equations near $a=0$ [22]. This should be worked out in more detail in order to decide, along the scheme given in section 5, whether the initial local singularity is avoided or not.

\section{Remarks.}

1. We have computed the expectation value of the analogon of the inverse scale factor with respect to kinematical coherent states whose peak follows the classical (singular) trajectory. One should do the same with respect to the matter degrees of freedom. However, it is clear from the outset that this will not change our result: the classical Friedmann equations in the gauge outlined above are

$$
3 H^{2}=8 \pi G\left(\frac{\pi^{2}}{2 a^{6}}+\frac{a^{3} V}{a^{3}}\right)+\Lambda-\frac{3 k}{a^{2}}, \quad \dot{\pi}=-a^{3} V^{\prime},
$$

where $H=\frac{\dot{a}}{a}$ is the Hubble function and $\pi=a^{3} \dot{\phi}$ the conjugate momentum. Consider the case of a potential which depends polynomially on $\phi$ but not exponentially. We want to solve (4.8) close to $a=0$. We now make the self-consistent assumption (to be checked) that $a^{3} V$ (and thus $a^{3} V^{\prime}$ ) vanishes in the limit $a \rightarrow 0$. Hence $\pi$ becomes constant close to $a=0$ according to the second equation in (4.8). Then we may neglect the potential $(V)$, cosmological $(\Lambda)$ and curvature $(k)$ term in the first equation as $a \rightarrow 0$ and thus we may solve $a(t) \propto t^{1 / 3}$ and $\phi(t) \propto \ln (t)$. Hence indeed $a^{3} \phi^{n} \propto t[\ln (t)]^{n}$ vanishes at $t=0$ and our assumption was correct. It follows that the expectation value with respect to coherent states, taking into account both matter and geometry, of the scalar contribution to the Hamiltonian constraint (2.7), whose homogeneous reduction is $\pi^{2} /\left(2 a^{3}\right)+a^{3} V$, is indeed non-singular even when the coherent states are evaluated along the exact classical trajectory, if and only if the expectation value of the analogon in (3.3) of $1 / a^{3}$ is bounded from above. This is precisely what we verified.

2. We have shown unboundedness of the inverse scale factor in LQG (with gauge group $S U(2)$ ) and did expectation value calculations thereof for an approximation to LQG using $U(1)^{3}$ instead of $S U(2)$. It is maybe worthwhile to mention that the inverse scale factor for that approximation is also unbounded on zero-volume eigenstates although one has to use graphs of valence at least five here which is due to the detailed difference between the non-Abelian group $S U(2)$ and the Abelian group $U(1)^{3}$. 
3. Note that the bound in formula (4.6) is completely general. It does not depend on the value of the classical connection $A$ that labels the coherent state, it only depends on the electric field $E$. Setting $E_{j}^{a}=0$ as appropriate for the isotropic singularity gives the upper bound (4.7). However, we may consider any other homogeneous model, for instance inhomogeneous ones such as Bianchi I (Kasner), Bianchi IX or Mixmaster Models (BKL scenario). In these cases the different components of the three metric vanish with different rates as we approach the singularity (or do not vanish at all or might even diverge, at least in the absence of matter). As we can see from the above general estimate, the important question is whether the quantity $p$ defined above, which is proportional to $E_{j}^{a}=\sqrt{\operatorname{det}(q)} e_{j}^{a}$, remains finite in all those cases. For instance, in the Kasner models with line element $\mathrm{d} s^{2}=-\mathrm{d} t^{2}+\sum_{a=1}^{3} t^{2 p_{a}}\left(\mathrm{~d} x^{a}\right)^{2}$ where the real parameters $p_{a}$ are subject to $\sum_{a} p_{a}=\sum_{a} p_{a}^{2}=1$ we find in the diagonal gauge $E_{j}^{a}=\delta_{j}^{a} t^{1-p_{a}}$ which stays bounded for $t \rightarrow 0$ because $p_{a} \leqslant 1$. In particular, contracting directions (with increasing $t$ ) are allowed. This covers all Kasner models and shows that the expectation value calculation performed here also hints at local singularity avoidance for at least some class of anisotropic models. Whether this holds for all homogeneous Bianchi models and also after matter is coupled is a task left to the reader.

\section{Scheme for determining the presence or absence of the initial singularity}

The computation of the previous section is very encouraging. Unfortunately, it does not yet prove that in LQG the big bang singularity or any other classical spacetime curvature singularity is quantum mechanically absent. As we have explained at length in the introduction, fundamentally, singularity avoidance must be formulated in terms of physical states ${ }^{24}$ and physical operators which has not been done so far even in $\mathrm{LQC}^{25}$ because the volume, the inverse scale factor and the value of the inflaton field etc are not gauge invariant quantities (they do not commute with e.g. the Hamiltonian constraint).

Fundamentally, what one would need to do in order to establish a singularity avoidance result is something along the following lines ${ }^{26}$ :

1. Ideally one should have sufficiently explicit knowledge about the physical Hilbert space $\mathcal{H}_{\text {Phys }}$ and physical states. An avenue towards this goal is the master constraint programme [17]. Note that physical states automatically solve the quantum Einstein equations. That means that the quantum gauge evolution [15], defined similarly as in [19] as the coefficients of the physical states when expanded into (spatially diffeomorphism invariant) spin network functions, is non-singular by definition thanks to the fact that operators like the inverse scale factor are densely defined in LQG. Moreover, in LQG there are physical states which are composed of spin network states that describe a sign flip with respect to the expectation value of the triad orientation sign operator [44] and thus may describe quantum geometries which include a pre-big bang in the sense of [19]. As we have already outlined in the introduction, the global singularity is avoided if and only if there are sufficiently many semiclassical of these physical states.

\footnotetext{
${ }^{24}$ The singularity effects A and B for the global singularity have indeed been discussed for solutions of the WheelerDeWitt equation in LQC. However, that does not grant that a sufficient number of them are normalizable or have non-vanishing norm in the physical inner product. Once again, formal solutions are not automatically physical states. ${ }^{25}$ In terms of the partial and complete observables discussed below, there is in general no relation between the spectra of the complete and partial observables [31]. Hence kinematical boundedness does not imply physical boundedness as far as the local singularity is concerned.

26 This is just a sketch of ideas, not a 'paradigm'.
} 
2. (In)famously, canonical quantum gravity, especially in the cosmological context, is a theory without a canonical Hamiltonian. However, one can construct physical Hamiltonians using the relational point of view [29, 30]. These are Dirac observables by construction. They are obtained by (i) using non-gauge invariant quantities $P, T$ where $P$ is called a partial observable and $T$ a collection of ${ }^{27}$ clock variables, (ii) computing the gauge flows (Hamiltonian evolution with respect to the Hamiltonian constraints ${ }^{28}$ ) $t \mapsto \alpha_{t}(P), \alpha_{t}(T)$, (iii) choosing parameters $\tau$ and solving the equations $\alpha_{t}(T)=\tau$ for $t$ and (iv) inserting those values of $t$ into $\alpha_{t}(P)$. The resulting object $P_{T}(\tau)$ has the physical interpretation of giving the value of $P$ at the moment (gauge parameter $t$ ) when $T$ has attained the value $\tau$. The function $P_{T}(\tau)$ is called the corresponding complete or Dirac observable. The physical Hamiltonians $H_{T}(\tau)$ are in general explicitly dependent on the physical time $\tau$ and are defined by $\left\{H_{T}(\tau), P_{T}(\tau)\right\}:=\mathrm{d} / \mathrm{d} \tau P_{T}(\tau)$ for all $P$. Of course there are many possible choices for $H_{T}$ depending on the choice of the 'clocks' $T$ (in GR we need an infinite number of those). In cosmology it would be nice to use the scale factor (total volume of space) as a clock. If a corresponding positive (or at least semibounded) and physical time independent Hamiltonian exists and can be defined as a self-adjoint operator on the physical Hilbert space then we may use it to define evolution on the physical Hilbert space.

3. If a physical Hamitonian is given, then we will need coherent states in $\mathcal{H}_{\text {Phys }}$ which are preserved by $\hat{H}_{T}$ for sufficiently long physical time. A general idea for how to do that has been given by Klauder [43] at least for systems with a finite number of degrees of freedom.

4. We are now in the position to address the issue about the local singularity: given a physical coherent state $\psi$ peaked, on scales large compared to the Planck scale, corresponding to $\tau=\tau_{0} \gg \tau_{P}$, on classical singular initial data, consider the map $\tau \mapsto\left\langle\psi, P_{T}\left(\tau-\tau_{0}\right) \psi\right\rangle_{\text {Phys }}=\left\langle\psi\left(\tau-\tau_{0}\right), \widehat{P_{T}\left(\tau_{0}\right)} \psi\left(\tau-\tau_{0}\right)\right\rangle_{\text {Phys }}$ with $\psi(\tau):=\mathrm{e}^{\mathrm{e} \tau \hat{H}_{T}} \psi$. Let $P$ be any classically singular quantity, say the matter energy density or a curvature scalar density (suitably smeared). Then the local singularity, with respect to $P$, is absent provided that $\left\langle\psi, \widehat{P_{T}\left(-\tau_{0}\right)} \psi\right\rangle_{\text {Phys }}$ is finite (assuming that $\tau=0$ corresponds to vanishing classical volume which will be the case if we choose $V$ as one of the clock variables).

Note that this ambitious scheme has not been followed even in the simplified context of LQC where, however, all the steps could presumably be carried out sufficiently explicitly; see [48] for first steps in that direction. In full LQG these steps cannot be carried out exactly because the theory is too complicated. Thus, in order to make progress one must use suitable approximation schemes. A possible starting point could be the following:

$1^{\prime}$. Using kinematical coherent states $\psi_{A, E}$ peaked on the constraint surface of the phase space we arrive at a subspace of the kinematical Hilbert space $\mathcal{H}_{\text {kin }}$ with the property that the constraints are satisfied approximately in the sense of expectation values, that is $\langle\psi, \hat{C} \psi\rangle \approx 0$. Denote that subspace by $\mathcal{H}_{\text {Phys }}^{\prime}{ }^{29}$. In systems where one can compare predictions by the exact $\mathcal{H}_{\text {Phys }}$ with those by $\mathcal{H}_{\text {Phys }}^{\prime}$ it is often the case that the qualitative answers do not differ much; see e.g. [46]. Since there are as many semiclassical kinematical states as classical initial data (classical spacetimes), global singularities

\footnotetext{
${ }^{27}$ In general one needs as many clock variables as there are constraints.

${ }^{28}$ There are also as many unphysical time parameters $t$ as there are constraints. In GR they become functions and are called lapse and shift.

${ }^{29}$ Alternatively, find an approximation method for the Master Constraint Programme (also using kinematical coherent states and path integral methods, see [17]).
} 
cannot be determined within this approximate scheme which uses the kinematical Hilbert space.

$2^{\prime}$. The formulae for $P_{T}(\tau)$ and $H_{T}$ are infinite power expansions in the 'parameters' $\tau-T$ [30]. Hence, close to the singularity, i.e. when $\tau \approx T=0$ (this has to be understood ultimately in terms of operators; we assume that the $T, \tau$ are chosen such that $T=\tau=0$ corresponds to the singularity) we may approximate those formulae by the first few terms and get approximate expressions $\hat{P}_{T}^{\prime}(\tau), \hat{H}_{T}^{\prime}$ that one can hope to handle. These approximations should be appropriate in order to answer qualitatively questions about the very early universe.

$3^{\prime}$. The coherent states of item $1^{\prime}$ should ideally be chosen as to be preserved by the approximate Hamiltonian determined in step $2^{\prime}$. If that is too difficult, peak the kinematical coherent states of step $1^{\prime}$ on classically singular initial data and then quantum evolve them with the (approximate) Hamiltonian $\hat{H}_{T}^{\prime}$, that is $\tau \mapsto \exp \left(i \tau \hat{H}_{T}^{\prime}\right) \psi_{A, E}$.

$4^{\prime}$. This step is identical to step 4 above with the obvious replacements.

Note that one could consider an even easier scheme: just take any set of kinematical coherent states not generated by any (approximate) physical Hamiltonian and consider the unphysical time evolution $t \mapsto \psi_{A_{0}, E_{0}}(t):=\psi_{A(t), E(t)}$ where $A(t), E(t)$ is a one parameter classical gauge evolution of the initial data under the flow of the corresponding Hamiltonian constraint (not of any physical Hamiltonian) $)^{30}$ with initial condition $A\left(t_{0}\right)=A_{0}, E\left(t_{0}\right)=E_{0}$ and $t_{0} \gg t_{P}$. Then take any quantity $P$ which is not necessarily a (approximate) Dirac observable and study $\left\langle\psi_{A(t), E(t)}, \hat{P} \psi_{A(t), E(t)}\right\rangle_{\text {kin }}$ in the limit $t \rightarrow 0$ corresponding to the classical singularity (in our case $A(t)$ diverges while $E(t)$ vanishes at $t=0)$. This is essentially what we did in the previous section where $P$ played the role of the inverse scale factor and the coherent states we used are those of [43]. While in this case we obtained a boundedness result, one may not draw any conclusions from that as the following example reveals: consider the Hamiltonian of the hydrogen atom. Let $\psi_{\vec{q}, \vec{p}}$ be the coherent states of the harmonic oscillator peaked at the point $(\vec{q}, \vec{p})$ in phase space. Let $\vec{q}(t), \vec{p}(t)$ be the trajectory of a classical electron falling radially into the proton from a distance large compared to the atomic radius where $t=0$ is the point of time when the electron reaches the centre. Then it is easy to see that $\left\langle\psi_{\vec{q}(t), \vec{p}(t)}, \hat{H} \psi_{\vec{q}(t), \vec{p}(t)}\right\rangle$ diverges as $t \rightarrow 0$. This is because the electron becomes infinitely fast at the centre and the expectation value of the kinetic term diverges (while the expectation value of the potential energy stays finite). In contrast, the expectation value $\left\langle\psi_{\vec{q}, \vec{p}}(t), \hat{H} \psi_{\vec{q}, \vec{p}}(t)\right\rangle=\left\langle\psi_{\vec{q}, \vec{p}}, \hat{H} \psi_{\vec{q}, \vec{p}}\right\rangle$ is finite where $\psi_{\vec{q}, \vec{p}}(t):=\exp (\mathrm{i} t \hat{H}) \psi_{\vec{q}, \vec{p}}$ is the quantum evolved state ${ }^{31}$. Thus, while $\psi_{\vec{q}(t), \vec{p}(t)}$ is nicely peaked on the classical trajectory, it does not correspond to the true quantum-mechanical time evolution. On the other hand, the true quantum-mechanical time evolution $\psi_{\vec{q}, \vec{p}}(t)$ usually does not stay peaked on the classical trajectory for sufficiently long time in order to qualify as a semiclassical state representing the classical evolution far away from the deep quantum regime closely enough, unless the coherent state is actually adapted to the Hamiltonian $\hat{H}$.

Summarizing, ideally the coherent states must be chosen according to the Hamiltonian in question in order to arrive at reliable predictions. If that is too hard, then at least the kinematical coherent state should be evolved quantum mechanically but only as long as it remains close to the classical trajectory for scales above which the classical theory is reliable. In contrast, the classically evolved kinematical semiclassical state not constructed according to the Hamiltonian is inappropriate in general which is why the calculation of section 4 is promising but inconclusive.

\footnotetext{
30 This corresponds to a specific choice of lapse and shift.

${ }^{31}$ Remarkably, the states $\psi_{\vec{q}, \vec{p}}(t)$ and $\psi_{\vec{q}(t), \vec{p}(t)}$ precisely coincide if and only if the underlying dynamics is that of a finite or infinite sum of uncoupled harmonic oscillators.
} 


\section{Conclusions}

In conclusion, the issue of the presence or absence of the initial singularity in full LQG remains open. The results of LQC are important, they are very promising, provide important consistency checks for LQG and produce new insights and ideas about LQG. However, as we hope to have demonstrated, they are so far inconclusive for LQG. Therefore sentences such as 'LQC is the cosmological sector of LQG' or 'in LQG the big bang is absent' [49] are misleading for the non-expert and should be used with due care.

As our detailed analysis reveals, one must pay attention to the QFT aspects of the full theory and cannot rely on the analysis of a quantum-mechanical toy model. This is, unfortunately, different from the case of the hydrogen atom where experiments confirmed from the outset that the quantum-mechanical toy model was close to the truth and that the more accurate QED should only give corrections. It may still be that LQC is qualitatively right but if that is the case then the mechanism for singularity resolution is very different in full LQG and much more elaborate, if LQG is a valid candidate for quantum gravity at all.

In order to resolve the issue about the initial singularity and other cosmological questions in full LQG we have proposed an ideal and approximate programme, highlighting the importance of having control over the physical Hilbert space and the physical observables. In a zerothorder step of that programme we found hints pointing at a resolution of the local initial singularity in LQG by a very different mechanism. But it is necessary to carry out all the steps before definite conclusions can be reached. Since this programme was not even completed in LQC, again the LQC model could be a good starting point to see where the ideal path leads us in a solvable situation. As far as the full theory is concerned we will only be able to follow the approximate path and we must learn how to control the corresponding approximation errors.

In summary, the investigation of the cosmological or black hole sector of LQG, about which the LQG inspired LQC model has taught us important first lessons, is an ambitious and fascinating research field which will require the joint effort of both cosmologists and quantum geometers in the close future.

\section{Acknowledgments}

It is our pleasure to thank Martin Bojowald for many clarifying comments and a careful reading of the manuscript. JB thanks the Gottlieb Daimler- and Karl Benz-Foundation and the DAAD (German Academic Exchange Service) for financial support. This work was supported in part by a grant from NSERC of Canada to the Perimeter Institute for Theoretical Physics.

\section{References}

[1] Rovelli C 2004 Quantum Gravity (Cambridge: Cambridge University Press) Thiemann T 2005 Modern Canonical Quantum General Relativity (Cambridge: Cambridge University Press) (Preprint gr-qc/0110034)

[2] Rovelli C 1998 Loop quantum gravity Living Rev. Rel. 11 (Preprint gr-qc/9710008)

Thiemann T 2003 Lectures on loop quantum gravity Lect. Notes Phys. 631 41-135 (Preprint gr-qc/0210094)

Ashtekar A and Lewandowski J 2004 Background independent quantum gravity: a status report Class. Quantum Grav. 21 R53 (Preprint gr-qc/0404018)

Smolin L 2004 An invitation to loop quantum gravity Preprint hep-th/0408048

[3] Brunetti R, Fredenhagen K and Verch R 2003 The generally covariant locality principle: a new paradigm for local quantum field theory Commun. Math. Phys. 237 31-68 (Preprint math-ph/0112041)

[4] Lauscher O and Reuter M 2002 Towards nonperturbative renormalizability of quantum Einstein gravity Int. $J$. Mod. Phys. A 17993 (Preprint hep-th/0112089) 
Lauscher O and Reuter M 2002 Is quantum gravity nonperturbatively renormalizable? Class. Quantum Grav. 19483 (Preprint hep-th/0110021)

[5] Bojowald M and Morales-Tecotl H 2004 Cosmological applications of loop quantum gravity Lect. Notes Phys. 646 421-62 (Preprint gr-qc/0306008)

[6] Halliwell J 1988 Derivation of the Wheeler-DeWitt equation from a path integral for minisuperspace models Phys. Rev. D 382468

[7] Sahlmann H 2002 When do measures on the space of connections support the triad operators of loop quantum gravity? Preprint gr-qc/0207112

Sahlmann H 2002 Some comments on the representation theory of the algebra underlying loop quantum gravity Preprint gr-qc/0207111

Sahlmann H and Thiemann T 2003 On the superselection theory of the Weyl algebra for diffeomorphism invariant quantum gauge theories Preprint gr-qc/0302090

Sahlmann H and Thiemann T 2003 Irreducibility of the Ashtekar-Isham-Lewandowski representation Preprint gr-qc/0303074

Okolow A and Lewandowski J 2003 Diffeomorphism covariant representations of the holonomy flux algebra Preprint gr-qc/0302059

Fleichhack C 2004 Representations of the Weyl algebra in quantum geometry Preprint math-ph/0407006

Lewandowski J, Okolow A, Sahlmann H and Thiemann T 2005 Uniqueness of diffeomorphism invariant states on holonomy-flux algebras Preprint gr-qc/0504147

[8] Ashtekar A and Isham C J 1992 Representations of the holonomy algebras of gravity and non-Abelean gauge theories Class. Quantum Grav. 91433 (Preprint hep-th/9202053)

Ashtekar A and Lewandowski J 1994 Representation theory of analytic holonomy $C^{\star}$ algebras Knots and Quantum Gravity ed J Baez (Oxford: Oxford University Press)

[9] Thirring W 1978 Lehrbuch der Mathematischen Physik vol 3 (Berlin: Springer)

[10] Acerbi F, Morchio G and Strocchi F 1993 Infrared singular fields and nonregular representations of CCR algebras J. Math. Phys. 34 899-914

[11] Thirring W and Narnhofer H 1992 Covariant QED without indefinite metric Rev. Math. Phys. SI1 197-211

[12] Thiemann T 2004 The LQG string: loop quantum gravity quantization of string theory: I. Flat target space Preprint hep-th/0401172

[13] Thiemann T 1996 Anomaly-free formulation of non-perturbative, four-dimensional Lorentzian quantum gravity Phys. Lett. B 380 257-64 (Preprint gr-qc/9606088)

Thiemann T 1998 Quantum spin dynamics (QSD) Class. Quantum Grav. 15 839-73 (Preprint gr-qc/9606089)

[14] Thiemann T 1998 Quantum spin dynamics: II. The kernel of the Wheeler-DeWitt constraint operator Class. Quantum Grav. 15 875-905 (Preprint gr-qc/9606090)

[15] Thiemann T 1998 Quantum spin dynamics: V. Quantum gravity as the natural regulator of the Hamiltonian constraint of matter quantum field theories Class. Quantum Grav. 15 1281-314 (Preprint gr-qc/9705019)

[16] Thiemann T 1998 Quantum spin dynamics: IV. 2+1 Euclidean quantum gravity as a model to test 3+1 Lorentzian quantum gravity Class. Quantum Grav. 15 1249-80 (Preprint gr-qc/9705018)

[17] Thiemann T 2003 The Phoenix Project: master constraint programme for loop quantum gravity Preprint gr-qc/0305080

Dittrich B and Thiemann T 2006 Testing the master constraint programme for loop quantum gravity: I. General framework Class. Quantum Grav. 23 1025-65 (Preprint gr-qc/0411138)

Dittrich B and Thiemann T 2006 Testing the master constraint programme for loop quantum gravity: II. Finite dimensional systems Class. Quantum Grav. 23 1067-88 (Preprint gr-qc/0411139)

Dittrich B and Thiemann T 2006 Testing the master constraint programme for loop quantum gravity: III. SL $(2, R)$ models Class. Quantum Grav. 23 1089-120 (Preprint gr-qc/0411140)

Dittrich B and Thiemann T 2006 Testing the master constraint programme for loop quantum gravity: IV. Free field theories Class. Quantum Grav. 23 1121-42 (Preprint gr-qc/0411141)

Dittrich B and Thiemann T 2006 Testing the master constraint programme for loop quantum gravity: V. Interacting field theories Class. Quantum Grav. 23 1143-62 (Preprint gr-qc/0411142)

[18] Thiemann T 1998 A length operator for canonical quantum gravity J. Math. Phys. 393372-92 (Preprint gr-qc/9606092)

[19] Bojowald M 2001 Absence of singularity in loop quantum cosmology Phys. Rev. Lett. 86 5227-30 (Preprint gr-qc/0102069)

[20] Bojowald M 2003 Homogeneous loop quantum cosmology Class. Quantum Grav. 202595 (Preprint gr-qc/0303073)

[21] Wald R M 1989 General Relativity (Chicago, IL: The University of Chicago Press)

[22] Bojowald M 2002 Inflation from quantum geometry Phys. Rev. Lett. 89261301 (Preprint gr-qc/0206054) 
[23] Bojowald M 2002 Isotropic loop quantum cosmology Class. Quantum Grav. 192717 (Preprint gr-qc/0202077)

[24] Husain V and Winkler O 2004 On singularity resolution in quantum gravity Phys. Rev. D 69084016 (Preprint gr-qc/0312094)

Husain V and Winkler O 2004 Quantum resolution of black hole singularities Preprint gr-qc/0410125

Hofmann S and Winkler O 2004 The spectrum of fluctuations in inflationary quantum cosmology Preprint astro-ph/0411124

[25] Kuchar K V and Ryan M P 1989 Is minisuperspace quantization valid? Taub in mixmaster Phys. Rev. D 40 3982-96

Sinha S and Hu B L 1991 Validity of the minisuperspace approximation: an example from interacting quantum field theory Phys. Rev. D 44 1028-37

Ishikawa A and Isse T 1993 The stability of minisuperspace Mod. Phys. Lett. A $83413-28$ (Preprint gr-qc/9308004)

Saremi O 2001 Is minisuperspace quantum gravity reliable? Preprint gr-qc/0101108

[26] Green D and Unruh W 2004 Difficulties with closed isotropic loop quantum cosmology Phys. Rev. D 70103502 (Preprint gr-qc/0408074)

Cartin D and Khanna G 2005 Absence of pre-classical solutions in Bianchi I loop quantum cosmology (Preprint gr-qc/0501016)

[27] Bojowald M 2005 Private communication

[28] Brunnemann J and Thiemann T 2006 Unboundedness of triad-like operators in loop quantum gravity Class. Quantum Grav. 23 1429-83 (Preprint gr-qc/0505033)

[29] Rovelli C 1991 Conceptual Problems in Quantum Gravity ed A Ashtekar and J Stachel (Boston, MA: Birhäuser) p 126

Rovelli C 1991 What is observable in classical and quantum gravity? Class. Quantum Grav. 81895

Rovelli C 2002 Partial observables Phys. Rev. D 65124013

[30] Dittrich B 2004 Partial and complete observables for Hamiltonian constrained systems Preprint gr-qc/0411013 Thiemann T 2004 Reduced phase space quantization and Dirac observables Preprint gr-qc/0411031

[31] Dittrich B and Thiemann T 2006 Facts and fiction about Dirac observables in (loop) quantum gravity in preparation

[32] Modesto L 2004 Disappearance of black hole singularity in quantum gravity Phys. Rev. D 70124009 (Preprint gr-qc/0407097)

Modesto L 2004 Kantowski-Sachs space-time in loop quantum gravity Preprint gr-qc/0411032

Modesto L 2005 Quantum gravitational collapse Preprint gr-qc/0504043

Ashtekar A and Bojowald M 2005 Black hole evaporation: a paradigm Preprint gr-qc/0504029

[33] Husain V and Winkler O 2004 On singularity resolution in quantum gravity Phys. Rev. D 69084016 (Preprint gr-qc/0312094)

Husain V and Winkler O 2004 Quantum resolution of black hole singularities Preprint gr-qc/0410125

Husain V and Winkler O 2004 Quantum black holes Preprint gr-qc/0412039

[34] Sahlmann H and Thiemann T 2006 Towards the QFT on curved spacetime limit of QGR: I. A general scheme Class. Quantum Grav. 23867 (Preprint gr-qc/0207030)

Sahlmann H and Thiemann T 2006 Towards the QFT on curved spacetime limit of QGR: II. A concrete implementation Class. Quantum Grav. 23909 (Preprint gr-qc/0207031)

[35] Thiemann T 1998 Closed formula for the matrix elements of the volume operator in canonical quantum gravity J. Math. Phys. 39 3347-71 (Preprint gr-qc/9606091)

Brunnemann J and Thiemann T 2004 Simplification of the spectral analysis of the volume operator in loop quantum gravity Preprint gr-qc/0405060

[36] De Pietri R 1997 Spin networks and recoupling in loop quantum gravity Preprint gr-qc/9701041

[37] Ashtekar A, Bojowald M and Lewandowski J 2003 Mathematical structure of loop quantum cosmology Adv. Theor. Math. Phys. 7233 (Preprint gr-qc/0304074)

[38] Gaul M and Rovelli C 2001 A generalized Hamiltonian constraint operator in loop quantum gravity and its simplest Euclidean matrix elements Class. Quantum Grav. 18 1593-624 (Preprint gr-qc/0011106)

[39] Bojowald M and Kastrup H 2000 Quantum symmetry reduction for diffeomorphism invariant theories of connections Class. Quantum Grav. 173009 (Preprint hep-th/9907042)

[40] Bombelli L 2000 Statistical Lorentzian geometry and the closeness of Lorentzian manifolds J. Math. Phys. 41 6944-58 (Preprint gr-qc/0002053)

Bombelli L, Corichi A and Winkler O 2004 Semiclassical quantum gravity: statistics of combinatorial Riemannian geometries Preprint gr-qc/0409006

[41] Thiemann T and Winkler O 2001 Gauge field theory coherent states (GCS): IV. Infinite tensor product and thermodynamic limit Class. Quantum Grav. 18 4997-5033 (Preprint hep-th/0005235) 
[42] Thiemann T 1998 Kinematical Hilbert spaces for fermionic and Higgs quantum field theories Class. Quantum Grav. 15 1487-512 (Preprint gr-qc/9705021)

Ashtekar A, Lewandowski J and Sahlmann H 2003 Polymer and Fock representations for a scalar field Class. Quantum Grav. 20 L11 (Preprint gr-qc/0211012)

[43] Thiemann T 2001 Quantum spin dynamics (QSD): VII. Symplectic structures and continuum lattice formulations of gauge field theories Class. Quantum Grav. 18 3293-338 (Preprint hep-th/0005232)

Thiemann T 2001 Gauge field theory coherent states (GCS): I. General properties Class. Quantum Grav. 18 2025-64 (Preprint hep-th/0005233)

Thiemann T and Winkler O 2001 Gauge field theory coherent states (GCS): II. Peakedness properties Class. Quantum Grav. 18 2561-636 (Preprint hep-th/0005237)

Thiemann T and Winkler O 2001 Gauge field theory coherent states (GCS): III. Ehrenfest theorems Class. Quantum Grav. 18 4629-81 (Preprint hep-th/0005234)

Sahlmann H, Thiemann T and Winkler O 2001 Coherent states for canonical quantum general relativity and the infinite tensor product extension Nucl. Phys. B 606 401-40 (Preprint gr-qc/0102038)

[44] Giesel K and Thiemann T 2005 Consistency check on volume and triad operator quantisation in loop quantum gravity: I Preprint gr-qc/0507036

Giesel K and Thiemann T 2005 Consistency check on volume and triad operator quantization in loop quantum gravity: II Preprint gr-qc/0507037

[45] Klauder J 2001 The current state of coherent states Preprint quant-ph/0110108

[46] Bolen B, Bombelli L and Corichi A 2004 Semiclassical states in quantum cosmology: Bianchi one coherent states Class. Quantum Grav. 21 4087-106 (Preprint gr-qc/0404004)

[47] Thiemann T 2002 Complexifier coherent states for canonical quantum general relativity Preprint gr-qc/0206037

[48] Noui K, Perez A and Vandersloot K 2005 On the physical Hilbert space of loop quantum cosmology Phys. Rev. D 71044025 (Preprint gr-qc/0411039)

[49] Tsujikawa S, Singh P and Maartens R 2004 Loop quantum gravity effects on inflation and the CMB Class. Quantum Grav. 21 5767-75 (Preprint astro-ph/0311015)

Bojowald M, Maartens R and Singh P 2004 Loop quantum gravity and the cyclic universe Phys. Rev. D 70083517 (Preprint hep-th/0407115)

Bojowald M, Maartens R and Singh P 2002 Inflation from quantum geometry Phys. Rev. Lett. 89261301 (Preprint gr-qc/0206054)

Bojowald M and Date G 2004 Quantum suppression of the generic chaotic behaviour to cosmological singularities Phys. Rev. Lett. 92071302 (Preprint gr-qc/0311003)

Bojowald M 2003 Initial conditions for a universe, essay awarded first prize in the gravity research foundation essay contest Gen. Rel. Grav. 35 1877-83 (Preprint gr-qc/0305069) 